\title{
Cyclic thermodynamic processes and entropy production
}

\section{Journal Article}

Author(s):

Abou Salem, Walid K.; Fröhlich, Jürg

Publication date:

2006

Permanent link:

https://doi.org/10.3929/ethz-b-000000346

Rights / license:

In Copyright - Non-Commercial Use Permitted

Originally published in:

Journal of Statistical Physics 126(3), https://doi.org/10.1007/s10955-006-9111-1 


\title{
Cyclic Thermodynamic Processes and Entropy Production
}

\author{
Walid K. Abou Salem and Jürg Fröhlich
}

Received January 8, 2006; accepted April 3, 2006

Published Online: December 15, 2006

\begin{abstract}
We study the time evolution of a periodically driven quantum-mechanical system coupled to several reservoirs of free fermions at different temperatures. This is a paradigm of a cyclic thermodynamic process. We introduce the notion of a Floquet Liouvillean as the generator of the dynamics of the coupled system on an extended Hilbert space. We show that the time-periodic state which the state of the coupled system converges to after very many periods corresponds to a zero-energy resonance of the Floquet Liouvillean. We then show that the entropy production per cycle is (strictly) positive, a property that implies Carnot's formulation of the second law of thermodynamics.
\end{abstract}

KEY WORDS: quantum statistical mechanics, Floquet theory, cyclic thermodynamic processes, entropy production

\section{INTRODUCTION}

During the past several years, there has been substantial progress in the program of deriving the fundamental laws of thermodynamics from nonequilibrium quantum statistical mechanics (see Refs. 3 and 4, for a synopsis). In this paper, we make a contribution to this program by studying Carnot's formulation of the second law of thermodynamics from the point of view of quantum statistical mechanics. For the sake of concreteness, we consider a periodically driven two-level quantum-mechanical system, $\Sigma$, coupled to $n \geq 2$ reservoirs of free fermions, $\mathcal{R}_{1}, \ldots, \mathcal{R}_{n}$. Our analysis can be generalized to a system composed of an arbitrary quantum-mechanical system with a finite-dimensional Hilbert space coupled to several reservoirs of (free) bosons or fermions at different

\footnotetext{
${ }^{1}$ Institut für Theoretische Physik, ETH-Hönggerberg CH-8093 Zürich, Switzerland; e-mail: walid@itp. phys.ethz.ch, juerg@itp.phys.ethz.ch
} 
temperatures. ${ }^{2}$ In order to study the time evolution of the coupled system, we extend Floquet theory for periodically driven quantum systems at zero temperature (see for example, Refs. 11, 25, 26) to apply to systems at positive temperatures.

In particular, we introduce an operator, the Floquet Liouvillean, generating the dynamics of the system on an extended Hilbert space, and we show that the timeperiodic state, which the state of the system converges to after very many periods, corresponds to a zero-energy resonance of the Floquet Liouvillean. We also prove (strict) positivity of entropy production per cycle, which amounts to Carnot's formulation of the second law of thermodynamics. For weak enough coupling of the small system, $\Sigma$, to the reservoirs, the time-periodic state corresponding to the zero-energy resonance can be expanded in powers of the coupling constant. This is of considerable practical importance, since it enables one to explicitly compute the degree of efficiency of the coupled system. Further discussion of the second law of thermodynamics and another proof of convergence to time-periodic states using methods of scattering theory will appear in Ref. 4. As far as we know, all previous investigations of Carnot's formulation of the second law of thermodynamics from the point of view of quantum statistical mechanics assumed that the coupling is switched on at time $t=t_{0}$, and then switched off at a later time $t=t_{0}+\tau$ (see Refs. 7, 22 and references therein). The novelty of our approach is to prove that the state of the coupled system converges to a time-periodic state with the same period as the one of the interaction, and that entropy production is (strictly) positive; (see also Refs. 10, 20, 21).

The organization of this paper is as follows. In Sec. 2, we recall some basic notions from quantum statistical mechanics, in particular, time-dependent perturbations of $C^{*}$-dynamical systems, relative entropy, and Carnot's formulation of the second law of thermodynamics. We also discuss sufficient conditions to prove strict positivity of entropy production per cycle. These conditions are satisfied in the concrete example we consider in the following Section. In Sec. 3, we discuss a concrete model, and we state the assumptions we make on the interaction between the small system, $\Sigma$, and the reservoirs, $\mathcal{R}_{1}, \ldots, \mathcal{R}_{n}$. In Sec. 4 , we introduce the Floquet Liouvillean, whose spectrum we study using complex spectral deformation techniques. In Sec. 5, we use results on the Floquet Liouvillean to prove convergence of the state of the coupled system to a time-periodic state. This is one of the main results of our paper. We then prove strict positivity of entropy production per cycle in Sec. 6, and we discuss how to compute the degree of efficiency of the coupled system for weak enough coupling. The main ingredients of our analysis are a concrete representation of the fermionic reservoirs

\footnotetext{
${ }^{2}$ Bosonic reservoirs are more difficult, technically, since the interaction term coupling $\Sigma$ to the bosonic reservoirs is generally an unbounded operator. However, one may readily extend the methods developed in Refs. 20, 21 to study the bosonic case.
} 
(Araki-Wyss representation), a spectral approach to cyclic thermodynamic processes using the so called Floquet Liouvillean, and complex spectral deformation techniques.

\section{GENERAL CONSIDERATIONS}

In this section, we recall some of the basic notions of quantum statistical mechanics, and we introduce the notion of time-periodic states. Although some of the material is standard, it is presented in this section in order to make our exposition reasonably self-contained.

In the algebraic formulation of quantum statistical mechanics, a physical system is described by a $C^{*}$ or $W^{*}$-dynamical system. Since we only consider fermionic reservoirs in this paper, we restrict our attention to the discussion of $C^{*}$ dynamical systems. However, our analysis can be generalized to $W^{*}$-dynamical systems; (see for example, Ref. 9, and also Refs. 20, 21).

A $C^{*}$-dynamical system is a pair $(\mathcal{O}, \alpha)$, where $\mathcal{O}$ is the kinematical algebra of the system, a $C^{*}$-algebra with identity, and $\alpha$, which specifies the time evolution, is a norm-continuous one-parameter group of $*$-automorphisms of $\mathcal{O}$. A physical state of the system is described by a positive, linear functional $\omega$, with $\omega(\mathbf{1})=1$. The set $E(\mathcal{O})$ of all states is a convex, weak-* compact subset of the dual $\mathcal{O}^{*}$.

Physically relevant states of (isolated) thermal reservoirs are assumed to be normal to equilibrium states characterized by the Kubo-Martin-Schwinger (KMS) condition. An equilibrium state of $(\mathcal{O}, \alpha)$ at inverse temperature $\beta, \omega_{\beta}$, is an $(\alpha, \beta)$-KMS state satisfying

$$
\omega_{\beta}\left(a \alpha^{t}(b)\right)=\omega_{\beta}\left(\alpha^{t-i \beta}(b) a\right),
$$

for all $a, b \in \mathcal{O}^{0}$, where $\mathcal{O}^{0}$ is norm-dense in $\mathcal{O}$.

We briefly recall the perturbation theory for $C^{*}$-dynamical systems; (for further details, see for example, Ref. 7). Let $\delta$ be the generator of $\alpha$, ie, $\alpha^{t}=$ $e^{t \delta}, t \in \mathbf{R}$. The domain of the derivation $\delta, \mathcal{D}(\delta)$, is a $*$-subalgebra of $\mathcal{O}$, and for all $a, b \in \mathcal{D}(\delta)$,

$$
\delta(a)^{*}=\delta\left(a^{*}\right), \delta(a b)=\delta(a) b+a \delta(b) .
$$

Consider the time-dependent family of perturbations, $\{g V(t)\}_{t \in \mathbf{R}}$ with selfadjoint elements $g V(t) \in \mathcal{O}$. Then $\alpha_{g}$, the perturbed time evolution, is a normcontinuous one-parameter family of $*$-automorphisms of $\mathcal{O}$ satisfying

$$
\frac{d}{d t} \alpha_{g}^{t}(a)=\alpha_{g}^{t}(\delta(a)+i g[V(t), a])
$$


and $\alpha_{g}^{0}(a)=a$, for all $a \in \mathcal{O}$. Explicity,

$$
\begin{aligned}
\alpha_{g}^{t}(a)= & \alpha^{t}(a)+\sum_{n \geq 1} i^{n} g^{n} \int_{0}^{t} d t_{1} \int_{0}^{t_{1}} d t_{2} \ldots \\
& \times \int_{0}^{t_{n-1}} d t_{n}\left[\alpha^{t_{n}}\left(V\left(t_{n}\right)\right), \ldots,\left[\alpha^{t_{1}}\left(V\left(t_{1}\right)\right), \alpha^{t}(a)\right], \ldots\right] .
\end{aligned}
$$

In the standard interaction picture,

$$
\alpha_{g}^{t}(a)=\Gamma_{g}^{t} \alpha^{t}(a) \Gamma_{g}^{t *},
$$

where $\Gamma_{g}$ is a unitary element of $\mathcal{O}$ which satisfies

$$
\frac{d}{d t} \Gamma_{g}^{t}=i \Gamma_{g}^{t} \alpha^{t}(g V(t))
$$

and $\Gamma_{g}^{0}=1 ;$ ie,

$$
\Gamma_{g}^{t}=\mathbf{1}+\sum_{n \geq 1} i^{n} g^{n} \int_{0}^{t} d t_{1} \int_{0}^{t_{1}} d t_{2} \cdots \int_{0}^{t_{n-1}} d t_{n} \alpha^{t_{n}}\left(V\left(t_{n}\right)\right) \cdots \alpha^{t_{1}}\left(V\left(t_{1}\right)\right) .
$$

Next, we discuss the notion of relative entropy and of entropy production. Assume that there exists a reference $C^{*}$-dynamics $\sigma_{\omega}$ on $\mathcal{O}$ and a state $\omega$ with the property that $\omega$ is an $\left(\sigma_{\omega},-1\right)$-KMS state. (Equivalently, at inverse temperature $\left.\beta \geq 0, \sigma_{\omega, \beta}^{t}=\sigma_{\omega}^{-t / \beta}\right)$. Let $\delta_{\omega}$ be the generator of $\sigma_{\omega}$, and let $\left(\mathcal{H}_{\omega}, \pi_{\omega}, \Omega_{\omega}\right)$ be the GNS representation of the kinematical algebra $\mathcal{O}$ associated to the state $\omega$. (For further discussion of the GNS construction see, for example, Ref. 7.)

A state $\eta \in E(\mathcal{O})$ is called $\omega$-normal if there exists a density matrix $\rho_{\eta}$ on $\mathcal{H}_{\omega}$, such that, for all $a \in \mathcal{O}$,

$$
\eta(a)=\operatorname{Tr}\left(\rho_{\eta} \pi_{\omega}(a)\right),
$$

where $\operatorname{Tr}$ is the trace over $\mathcal{H}_{\omega}$. We will denote by $\mathcal{N}_{\omega}$ the set of all $\omega$-normal states in $E(\mathcal{O})$.

For a state $\eta \in \mathcal{N}_{\omega}$, which might be time-dependent, denote by $\operatorname{Ent}(\eta \mid \omega)$ the relative entropy of Araki. $(1,8)$ For finite systems,

$$
\operatorname{Ent}(\eta \mid \omega)=-\operatorname{Tr}(\eta \log \omega-\eta \log \eta) .
$$

If $\eta \notin \mathcal{N}_{\omega}$, set $\operatorname{Ent}(\eta \mid \omega)=+\infty .^{3}$

For a self-adoint perturbation $g V(t) \in \mathcal{D}\left(\delta_{\omega}\right)$ of the dynamical system, as discussed above, we define the rate of entropy production in a state $\eta \in E(\mathcal{O})$ relative to a reference state $\omega$ as

$$
\operatorname{Ep}(\eta):=\eta\left(\delta_{\omega}(g V(t))\right)
$$

see for example, Refs. 7 and 17.

${ }^{3}$ Note the choice of the sign of relative entropy. 
It is instructive to see how one obtains this expression for entropy production as the thermodynamic limit of quantities referring to finitely extended reservoirs, Ref. 5. Consider a quantum system composed of a small system, $\Sigma$, coupled to $n$ reservoirs, $\mathcal{R}_{1}, \ldots, \mathcal{R}_{n}$, at inverse temperatures $\beta_{1}, \ldots, \beta_{n}$, respectively. We first treat the reservoirs as finitely extended systems and then take the thermodynamic limit of suitable quantities. The total Hamiltonian of the finite coupled system is

$$
H(t)=H^{\Sigma}+\sum_{i=1}^{n} H^{\mathcal{R}_{i}}+g V(t),
$$

where $H^{\Sigma}$ is the Hamiltonian of the uncoupled small system, $H^{\mathcal{R}_{i}}$ is the Hamiltonian of the $i$ th uncoupled reservoir, and $g V(t)$ is the interaction term coupling $\Sigma$ to the reservoirs. Let the reference state of the reservoirs be $\omega^{\mathcal{R}}$ with corresponding density matrix $\rho^{\mathcal{R}}$ given by

$$
\rho^{\mathcal{R}}=\rho^{\mathcal{R}_{1}} \otimes \cdots \otimes \rho^{\mathcal{R}_{n}},
$$

where $\rho^{\mathcal{R}_{i}}$ is the density matrix corresponding to the equilibrium state of the reservoir $\mathcal{R}_{i}$ at inverse temperature $\beta_{i}$, which is given by

$$
\rho^{\mathcal{R}}=\frac{e^{-\beta_{i} H^{\mathcal{R}_{i}}}}{\operatorname{Tr}_{\mathcal{R}_{i}}\left(e^{-\beta_{i} H^{\mathcal{R}_{i}}}\right)},
$$

where $T r_{\mathcal{R}_{i}}$ is the trace over the Hilbert space of the reservoir $\mathcal{R}_{i}$.

Define $\omega_{t}:=\omega \circ \alpha_{g}^{t}$, where $\omega$ is the initial $\left(\sigma_{\omega},-1\right)$-KMS state, and let $\rho^{\omega}$ be the density matrix corresponding to $\omega$. For a finite system,

$$
\begin{aligned}
\operatorname{Ent}\left(\omega_{t} \mid \omega^{\mathcal{R}}\right) & =-\operatorname{Tr}\left(\rho^{\omega_{t}} \log \rho^{\mathcal{R}}\right)+\operatorname{Tr}\left(\rho^{\omega_{t}} \log \rho^{\omega_{t}}\right) \\
& =-\operatorname{Tr}\left(\rho^{\omega_{t}} \log \rho^{\mathcal{R}}\right)+\operatorname{Tr}\left(\rho^{\omega_{t}} \log \rho^{\omega}\right),
\end{aligned}
$$

and hence

$$
\frac{d}{d t} \operatorname{Ent}\left(\omega_{t} \mid \omega^{\mathcal{R}}\right)=i \operatorname{Tr}\left(\left[H(t), \rho^{\omega_{t}}\right] \log \rho^{\mathcal{R}}\right) .
$$

By cyclicity of the trace we have that

$$
\begin{aligned}
\frac{d}{d t} \operatorname{Ent}\left(\omega_{t} \mid \omega^{\mathcal{R}}\right) & =i \sum_{i=1}^{n} \beta_{i} \operatorname{Tr}\left(\rho^{\omega_{t}}\left[H(t), H^{\mathcal{R}_{i}}\right]\right) \\
& =i \sum_{i=1}^{n} \beta_{i} \operatorname{Tr}\left(\rho^{\omega_{t}}\left[g V(t), H^{\mathcal{R}_{i}}\right]\right) \\
& =\omega \circ \alpha_{g}^{t}\left(\delta_{\omega}(g V(t))\right),
\end{aligned}
$$


where we have used in the last equation that $\delta_{\omega}=-\sum_{i=1}^{n} \beta_{i} \delta_{i}$, and $\delta_{i}=i\left[H^{\mathcal{R}_{i}}, \cdot\right]$. Note that the thermodynamic limit of the entropy production rate is well-defined.

One may relate the entropy production to the heat flux from the reservoirs. The heat flux from reservoir $\mathcal{R}_{i}, i=1, \ldots, n$, at time $t$ is

$$
\begin{aligned}
\Phi^{\mathcal{R}_{i}}(t): & =-\frac{d}{d t} \omega \circ \alpha_{g}^{t}\left(H^{\mathcal{R}_{i}}\right) \\
& =-i \omega\left(\alpha_{g}^{t}\left(\left[g V(t), H^{\mathcal{R}_{i}}\right]\right)\right) \\
& =\omega \circ \alpha_{g}^{t}\left(\delta_{i}(g V(t))\right) .
\end{aligned}
$$

It follows that

$$
\sum_{i=1}^{n} \beta_{i} \Phi^{\mathcal{R}_{i}}(t)=-E p\left(\omega \circ \alpha_{g}^{t}\right),
$$

which, for $n=1$ and reversible processes, is a familiar equation.

We now give a definition of a time-periodic state in case of a time-periodic coupling.

\subsection{Time-periodic State ${ }^{4}$}

Assume that the perturbation $g V(t)$ is time periodic with period $\tau$, and is norm-differentiable, for $t>0$. For $s \in[0, \tau)$, define the time-periodic state $\omega_{g, s}^{+}$ as

$$
\omega_{g, s}^{+}:=\lim _{n \rightarrow \infty} \omega \circ \alpha_{g}^{n \tau+s} .
$$

Note that it follows from this definition of a time-periodic state that,

$$
\omega_{g, s}^{+} \circ \alpha_{g}^{\tau}=\omega_{g, s}^{+} .
$$

We will show in Sec. 5 that this state is related to a zero-energy resonance of the so called Floquet Liouvillean.

Next, we exhibit a connection to Carnot's formulation of the second law of thermodynamics. Consider a cyclic thermodynamic process in which $\Sigma$ is coupled to two reservoirs, $\mathcal{R}_{1}$ and $\mathcal{R}_{2}$, at temperatures $T_{1}$ and $T_{2}$, respectively, with $T_{1}>T_{2}$. Reservoir 1 acts as a heat source and reservoir 2 as a heat sink. Recall that the generator of the free dynamics of reservoir $\mathcal{R}_{i}$ is $\delta_{i}, i=1,2$. Since $V(t)$ is norm

\footnotetext{
${ }^{4} \mathrm{~A}$ weaker definition of a time-periodic state is

$$
\omega_{g, s}^{+}:=\lim _{n \rightarrow \infty} \frac{1}{n \tau} \int_{0}^{n \tau} d t \omega \circ \alpha_{g}^{t+s} .
$$
}


differentiable, for $t>0$, it follows that

$$
\frac{d}{d t} \delta_{i}\left(\Gamma_{g}^{t}\right)=\delta_{i}\left(\frac{d}{d t} \Gamma_{g}^{t}\right),
$$

for $i=1,2$. Using the fact that $\delta_{i}, i=1,2$, is a $*$-derivation which commutes with $\alpha^{t}$ and Eq. (3), it follows that

$$
\frac{d}{d t} \omega\left(\Gamma_{g}^{t} \delta_{i}\left(\Gamma_{g}^{t *}\right)\right)=i \omega \circ \alpha_{g}^{t}\left(\delta_{i}(g V(t))\right)
$$

Therefore, the heat energy flowing from reservoir $\mathcal{R}_{i}$ into system $\Sigma$ during the time interval $[0, t]$ is

$$
\begin{aligned}
\Delta_{0}^{t} Q_{i} & =-\int_{0}^{t} d t^{\prime} \omega \circ \alpha_{g}^{t^{\prime}}\left(\delta_{i}\left(g V\left(t^{\prime}\right)\right)\right) \\
& =-i \omega\left(\Gamma_{g}^{t} \delta_{i}\left(\Gamma_{g}^{t *}\right)\right)
\end{aligned}
$$

$i=1,2$. Moreover, integrating (8), we have

$$
\beta_{1} \Delta_{0}^{t} Q_{1}+\beta_{2} \Delta_{0}^{t} Q_{2}=-\operatorname{Ent}\left(\omega \circ \alpha_{g}^{t} \mid \omega\right) \leq 0,
$$

since the relative entropy $\operatorname{Ent}\left(\omega \circ \alpha_{g}^{t} \mid \omega\right) \geq 0 .{ }^{5}$

Now define the heat flow per cycle from each reservoir $\mathcal{R}_{i}$ into $\Sigma$ as

$$
\Delta Q_{i}:=\lim _{n \rightarrow \infty}\left[Q_{i}((n+1) \tau)-Q_{i}(n \tau)\right], i=1,2 .
$$

We assume that the system converges to a time-periodic state, and that during every cycle, it performs work, ie,

$$
\Delta A=\Delta Q_{1}+\Delta Q_{2} \geq 0 .
$$

It follows from the definite sign of relative entropy and the existence of the time-periodic limit that the entropy production per cycle is nonnegative,

$$
\Delta E n t=\int_{0}^{\tau} d t \omega_{g, t}^{+}\left(\delta_{\omega}(g V(t))\right)=-\left(\beta_{1} \Delta Q_{1}+\beta_{2} \Delta Q_{2}\right) \geq 0 .
$$

The fact that $\beta_{1} \leq \beta_{2}$, (14) and (15), imply that $\Delta Q_{1} \geq 0$. It then follows that the degree of efficiency,

$$
\begin{aligned}
\eta & :=\frac{\Delta A}{\Delta Q_{1}} \\
& =\frac{\Delta Q_{1}+\Delta Q_{2}}{\Delta Q_{1}} \\
& \leq \frac{T_{1}-T_{2}}{T_{1}}=: \eta^{\text {Carnot }}
\end{aligned}
$$

\footnotetext{
${ }^{5}$ This follows from a general trace inequality, see for example, Ref. 7.
} 
which is nothing but Carnot's formulation of the second law of thermodynamics. In certain situations, one can show that

$$
\lim _{n \rightarrow \infty}\left[\operatorname{Ent}\left(\omega \circ \alpha_{g}^{(n+1) \tau} \mid \omega\right)-\operatorname{Ent}\left(\omega \circ \alpha_{g}^{n \tau} \mid \omega\right)\right]>0,
$$

which holds for the model we consider in this paper, and hence $\eta<\eta^{\text {Carnot }}$. The following proposition states sufficient conditions for inequality (19) to hold.

Proposition 2.1. For $t \in \mathbf{R}^{+}$, let $s:=t \bmod \tau$. Suppose that

(a) $\omega_{g, s}^{+} \notin \mathcal{N}_{\omega}$, and that

(b) $\sup _{T \in \mathbf{R}_{+}}\left|\int_{0}^{T} d t\left\{\omega_{g, t \bmod \tau}^{+}\left(\delta_{\omega}(g V(t))\right)-\omega \circ \alpha_{g}^{t}\left(\delta_{\omega}(g V(t))\right)\right\}\right|<C$ where $C$, is a finite, nonnegative constant.

Then $\operatorname{Ep}\left(\omega_{g, s}^{+}\right)>0$.

Proof: Suppose that $E p\left(\omega_{g, s}^{+}\right)=0$. Then

$$
\begin{aligned}
\operatorname{Ent}\left(\omega \circ \alpha_{g}^{t} \mid \omega\right) & =\int_{0}^{t} d t^{\prime} \omega \circ \alpha_{g}^{t^{\prime}}\left(\delta_{\omega}\left(g V\left(t^{\prime}\right)\right)\right) \\
& =\int_{0}^{t} d t^{\prime}\left\{\omega \circ \alpha_{g}^{t^{\prime}}\left(\delta_{\omega}\left(g V\left(t^{\prime}\right)\right)\right)-\omega_{g, t^{\prime} m o d}^{+}\left(\delta_{\omega}\left(g V\left(t^{\prime}\right)\right)\right)\right\} \\
& \leq C .
\end{aligned}
$$

In particular,

$$
\operatorname{Ent}\left(\omega_{g, s}^{+} \mid \omega\right)=\lim _{n \rightarrow \infty} \operatorname{Ent}\left(\omega \circ \alpha_{g}^{n \tau+s} \mid \omega\right) \leq C .
$$

Let $\mathcal{M}=\pi_{\omega}(\mathcal{O})^{\prime \prime}$, the double commutant of $\pi_{\omega}(\mathcal{O})$, and let $\mathcal{M}_{*}$ be its predual. The set of all states $\gamma \in \mathcal{N}_{\omega}$ such that $\operatorname{Ent}(\gamma \mid \omega) \leq C$ is $\sigma\left(\mathcal{M}_{*}, \mathcal{M}\right)$-compact (see Refs. 7 and 8). It follows that $\omega_{g, s}^{+} \in \mathcal{N}_{\omega}$, which contradicts assumption (a).

\subsection{Summary of Main Results}

Before specifying the concrete model we study, we briefly describe the main results of this paper, deferring precise statements and proofs to subsequent sections. One key result of this paper is transposing the problem of proving convergence to a time-periodic state to a spectral problem by introducing the so called Floquet Liouvillean (Sec. 4). In Sec. 5, Theorem 5.1, we show that the time-periodic state to which the state of the coupled system converges after very many periods is related to a zero-energy resonance of the Floquet Liouvillean. We also establish strict positivity of entropy production per cycle in the time-periodic state (Sec. 6, Theorem 6.3). In the case of two reservoirs,positivity of entropy 
production implies Carnot's formulation of the second law of thermodynamics,

$$
\eta<\eta^{\text {Carnot }} \text {. }
$$

Our analysis also has some quantitative implications: At weak coupling, the time-periodic state is analytic in the coupling constant, and hence one can calculate the entropy production per cycle perturbatively. This leads to a perturbative calculation of the degree of efficiency when the system is operated as a heat engine.

\section{THE MODEL}

As an example, we consider a two-level quantum system $\Sigma$ coupled to $n$ reservoirs, $\mathcal{R}_{1}, \ldots, \mathcal{R}_{n}, n \geq 2$, of free fermions in thermal equilibrium at inverse temperatures $\beta_{1}, \ldots, \beta_{n}$, and chemical potentials $\mu_{1}, \ldots, \mu_{n}{ }^{6}$

\subsection{The Small System}

The kinematical algebra of $\Sigma$ is $\mathcal{O}^{\Sigma}=\mathcal{M}\left(\mathbf{C}^{2}\right)$, the algebra of complex $2 \times 2$ matrices over the Hilbert space $\mathcal{H}^{\Sigma}=\mathbf{C}^{2}$. Its Hamiltonian is given by $H^{\Sigma}=\omega_{0} \sigma_{3}$, where $\sigma_{i}, i=1,2,3$, are the Pauli matrices. When the system $\Sigma$ is not coupled to the reservoirs, its dynamics in the Heisenberg picture is given by

$$
\alpha_{\Sigma}^{t}(a):=e^{i H^{\Sigma} t} a e^{-i H^{\Sigma} t},
$$

for $a \in \mathcal{O}^{\Sigma}$.

A physical state of the small system is described by a density matrix $\rho_{\Sigma}$. The operator $\kappa_{\Sigma}=\rho_{\Sigma}^{1 / 2}$ belongs to the space of Hilbert-Schmidt operators, which is isomorphic to $\mathcal{H}^{\Sigma} \otimes \mathcal{H}^{\Sigma}$. Two commuting representations of $\mathcal{O}^{\Sigma}$ on $\mathcal{H}^{\Sigma} \otimes \mathcal{H}^{\Sigma}$ are given by

$$
\begin{aligned}
& \pi_{\Sigma}(a):=a \otimes \mathbf{1}^{\Sigma}, \\
& \pi_{\Sigma}^{\#}(a):=\mathbf{1}^{\Sigma} \otimes C^{\Sigma} a C^{\Sigma},
\end{aligned}
$$

where $C^{\Sigma}$ is an antiunitary involution on $\mathcal{H}^{\Sigma}$ corresponding to complex conjugation; (see for example, Ref. 6).

The generator of the free dynamics on the Hilbert space $\mathcal{H}^{\Sigma} \otimes \mathcal{H}^{\Sigma}$ is the standard Liouvillean

$$
\mathcal{L}^{\Sigma}=H^{\Sigma} \otimes \mathbf{1}^{\Sigma}-\mathbf{1}^{\Sigma} \otimes H^{\Sigma}
$$

The spectrum of $\mathcal{L}^{\Sigma}$ is $\sigma\left(\mathcal{L}^{\Sigma}\right)=\left\{-2 \omega_{0}, 0,2 \omega_{0}\right\}$, with double degeneracy at zero.

\footnotetext{
${ }^{6}$ For the sake of simplicity of exposition, we set the chemical potentials of the reservoirs to be equal in the subsequent sections.
} 
Let $\omega^{\Sigma}$ be the initial state of the small system $\Sigma$ with corresponding vector $\Omega^{\Sigma} \in \mathcal{H}^{\Sigma} \otimes \mathcal{H}^{\Sigma}$. The modular operator associated with $\omega^{\Sigma}$ is $\Delta^{\Sigma}=\omega^{\Sigma} \otimes \bar{\omega}^{\Sigma^{-1}}$, and the modular conjugation operator, $J^{\Sigma}$, is given by

$$
J^{\Sigma}(\phi \otimes \psi)=\bar{\psi} \otimes \bar{\phi},
$$

for $\phi, \psi \in \mathcal{H}^{\Sigma}$. If $\omega_{\Sigma}$ corresponds to the trace state, then $\Delta^{\Sigma}=\mathbf{1}^{\Sigma} \otimes \mathbf{1}^{\Sigma}$.

\subsection{The Reservoirs}

Each thermal reservoir is formed of free fermions. It is infinitely extended and dispersive. We assume that the Hilbert space of a single fermion is $\mathbf{h}=L^{2}\left(\mathbf{R}^{+}, m(u) d u ; \mathcal{B}\right)$, where $\mathcal{B}$ is an auxiliary Hilbert space, and $m(u) d u$ is a measure on $\mathbf{R}^{+}$. We also assume that the single-fermion Hamiltonian, $h$, corresponds to the operator of multiplication by $u \in \mathbf{R}^{+}$. For instance, for reservoirs formed of nonrelativistic fermions in $\mathbf{R}^{3}$, the auxiliary Hilbert space $\mathcal{B}$ is $L^{2}\left(S^{2}, d \sigma\right)$, where $S^{2}$ is the unit sphere in $\mathbf{R}^{3}, d \sigma$ is the uniform measure on $S^{2}$, and $u=|\vec{k}|^{2}$, where $\vec{k} \in \mathbf{R}^{3}$ is the particle's momentum. In the latter case, the measure on $\mathbf{R}^{+}$is choosen to be $m(u) d u=\frac{1}{2} \sqrt{u} d u$. For the sake of concreteness, we will consider $\mathcal{B}=L^{2}\left(S^{d-1}, d \sigma\right), d>2$, in the sequel.

Let $b$ and $b^{*}$ be the annihilation-and creation operators on the Fermionic Fock space $\mathcal{F}\left(L^{2}\left(\mathbf{R}^{+} ; \mathcal{B}\right)\right)$. They satisfy the CAR

$$
\begin{aligned}
& \left\{b^{\#}(f), b^{\#}(g)\right\}=0, \\
& \left\{b(f), b^{*}(g)\right\}=(f, g) \mathbf{1},
\end{aligned}
$$

where $b^{\#}$ stands for $b$ or $b^{*}, f, g \in L^{2}\left(\mathbf{R}^{+} ; \mathcal{B}\right)$, and $(\cdot, \cdot)$ denotes the scalar product in $L^{2}\left(\mathbf{R}^{+} ; \mathcal{B}\right)$. Moreover, let $\Omega^{\mathcal{R}}$ denote the vacuum state in $\mathcal{F}\left(L^{2}\left(\mathbf{R}^{+} ; \mathcal{B}\right)\right)$.

The kinematical algebra, $\mathcal{O}^{\mathcal{R} i}$, of the $i$ th reservoir $\mathcal{R}_{i}, i=1, \ldots, n$, is generated by $b^{\#}$ and the identity $\mathbf{1}^{\mathcal{R}_{i}}$. The free dynamics of each reservoir (before the systems are coupled) is given by

$$
\alpha_{\mathcal{R}_{i}}^{t}\left(b_{i}^{\#}(f)\right)=b_{i}^{\#}\left(e^{i t u} f\right),
$$

for $i=1, \ldots, n, f \in L^{2}\left(\mathbf{R}^{+} ; \mathcal{B}\right)$. For a nonzero chemical potential, $\mu_{i}$, of reservoir $\mathcal{R}_{i}$, an auxiliary free dynamics is generated by $\tilde{H}^{\mathcal{R}_{i}}=d \Gamma_{i}\left(h-\mu_{i}\right)$; see for example, Ref. 7.

The $\left(\alpha_{\mathcal{R}_{i}}^{t}, \beta_{i}, \mu_{i}\right)$-KMS state, $\omega^{\mathcal{R}_{i}}$, of each reservoir $\mathcal{R}_{i}, i=1, \ldots, n$, at inverse temperature $\beta_{i}$ and chemical potential $\mu_{i}$, is the gauge invariant, quasifree state uniquely determined by the two-point function

$$
\omega^{\mathcal{R}_{i}}\left(b_{i}^{*}(f) b_{i}(f)\right)=\left(f, \rho_{\beta_{i}, \mu_{i}}(\cdot) f\right),
$$

where $\rho_{\beta_{i}, \mu_{i}}(u):=\frac{1}{e^{\beta_{i}\left(u-\mu_{i}\right)}+1}$. 
Next, we introduce $\mathcal{F}_{i}^{A W}:=\mathcal{F}^{\mathcal{R}_{i}}\left(L^{2}\left(\mathbf{R}^{+} ; \mathcal{B}\right)\right) \otimes \mathcal{F}^{\mathcal{R}_{i}}\left(L^{2}\left(\mathbf{R}^{+} ; \mathcal{B}\right)\right)$, the GNS Hilbert space for the Araki-Wyss representation of each fermionic reservoir $\mathcal{R}_{i}$ associated with the state $\omega^{\mathcal{R}_{i}}$. (2) Let $\tilde{b}_{i}$ and $\tilde{b}_{i}^{*}$ denote the annihilation- and creation operators on $\mathcal{F}^{\mathcal{R}_{i}}\left(L^{2}\left(\mathbf{R}^{+} ; \mathcal{B}\right)\right)$ satisfying the CAR, and denote by $\Omega^{\mathcal{R}_{i}}$ the vacuum state in $\mathcal{F}^{\mathcal{R}_{i}}\left(L^{2}\left(\mathbf{R}^{+} ; \mathcal{B}\right)\right)$, with $\tilde{b}_{i} \Omega^{\mathcal{R}_{i}}=0$. The Araki-Wyss representation, $\pi_{i}$, of the kinematical algebra $\mathcal{O}^{\mathcal{R}_{i}}, i=1, \ldots, n$, on $\mathcal{F}_{i}^{A W}$ is given by

$$
\begin{aligned}
& \pi_{i}\left(b_{i}(f)\right):=\tilde{b}_{i}\left(\sqrt{1-\rho_{\beta_{i}, \mu_{i}}} f\right) \otimes \mathbf{1}^{\mathcal{R}_{i}}+(-1)^{N_{i}} \otimes \tilde{b}_{i}^{*}\left(\sqrt{\rho_{\beta_{i}, \mu_{i}}} \bar{f}\right), \\
& \pi_{i}^{\#}\left(b_{i}(f)\right):=\tilde{b}_{i}^{*}\left(\sqrt{\rho_{\beta_{i}, \mu_{i}}} f\right)(-1)^{N_{i}} \otimes(-1)^{N_{i}}+\mathbf{1}^{\mathcal{R}_{i}} \otimes(-1)^{N_{i}} \tilde{b}_{i}\left(\sqrt{1-\rho_{\beta_{i}, \mu_{i}}} \bar{f}\right)
\end{aligned}
$$

where $N_{i}=d \Gamma_{i}(1)$ is the particle number operator for reservoir $\mathcal{R}_{i}$. Furthermore, $\Omega^{\mathcal{R}_{i}} \otimes \Omega^{\mathcal{R}_{i}} \in \mathcal{F}_{i}^{A W}$ corresponds to the equilibrium KMS state $\omega^{\mathcal{R}_{i}}$ of reservoir $\mathcal{R}_{i}$.

The free dynamics on the GNS Hilbert space $\mathcal{F}_{i}^{A W}$ of each reservoir $\mathcal{R}_{i}$ is generated by the standard Liouvillean $\mathcal{L}^{\mathcal{R}_{i}}$. The modular operator associated with $\left(\mathcal{O}^{\mathcal{R}_{i}}, \omega^{\mathcal{R}_{i}}\right)$ is given by

$$
\Delta^{\mathcal{R}_{i}}=e^{-\beta_{i} \mathcal{L}^{\mathcal{R}_{i}}},
$$

and the modular conjugation is given by

$$
J^{\mathcal{R}_{i}}(\Psi \otimes \Phi)=(-1)^{N_{i}\left(N_{i}-1\right) / 2} \bar{\Phi} \otimes(-1)^{N_{i}\left(N_{i}-1\right) / 2} \bar{\Psi}
$$

for $\Psi, \Phi \in \mathcal{F}_{i}^{A W}$; (see, for example, Ref. 7).

In order to apply the complex translation method developed in (Refs. 1416), we map $\mathcal{F}_{i}^{A W}:=\mathcal{F}^{\mathcal{R}_{i}}\left(L^{2}\left(\mathbf{R}^{+} ; \mathcal{B}\right)\right) \otimes \mathcal{F}^{\mathcal{R}_{i}}\left(L^{2}\left(\mathbf{R}^{+} ; \mathcal{B}\right)\right)$ to $\mathcal{F}^{\mathcal{R}_{i}}\left(L^{2}(\mathbf{R} ; \mathcal{B})\right)$ using the isomorphism between $L^{2}\left(\mathbf{R}^{+} ; \mathcal{B}\right) \oplus L^{2}\left(\mathbf{R}^{+} ; \mathcal{B}\right)$ and $L^{2}(\mathbf{R} ; \mathcal{B})$. To every $f \in L^{2}\left(\mathbf{R}^{+} ; \mathcal{B}\right)$, we associate functions $f_{\beta, \mu}, f_{\beta, \mu}^{\#} \in L^{2}(\mathbf{R} ; \mathcal{B})$ by setting

$$
f_{\beta, \mu}(u, \sigma):=\left\{\begin{array}{ll}
\sqrt{m(u)} \sqrt{1-\rho_{\beta, \mu}(u)} f(u, \sigma), & u \geq 0 \\
\sqrt{m(-u)} \sqrt{\rho_{\beta, \mu}(-u)} \bar{f}(-u, \sigma), & u<0
\end{array},\right.
$$

and

$$
\begin{aligned}
f_{\beta, \mu}^{\#}(u, \sigma): & =\left\{\begin{array}{l}
\sqrt{m(u)} i \sqrt{\rho_{\beta, \mu}(u)} f(u, \sigma), u \geq 0, \\
\sqrt{m(-u)} i \sqrt{1-\rho_{\beta, \mu}(-u)} \bar{f}(-u, \sigma), u<0,
\end{array}\right. \\
& =i \bar{f}_{\beta, \mu}(-u, \sigma),
\end{aligned}
$$

where $m(u) d u$ is the measure on $\mathbf{R}^{+}$, see Eq. (28). (For a discussion of this map, see Appendix)

Let $a_{i}$ and $a_{i}^{*}$ be the annihilation and creation operators on $\mathcal{F}^{\mathcal{R}_{i}}\left(L^{2}(\mathbf{R}, d u ; \mathcal{B})\right)$. Then

$$
\pi_{i}\left(b_{i}^{\#}(f)\right) \rightarrow a_{i}^{\#}\left(f_{\beta_{i}, \mu_{i}}\right)
$$




$$
\begin{aligned}
& \pi_{i}^{\#}\left(b_{i}^{\#}(f)\right) \rightarrow i(-1)^{N_{i}} a_{i}^{\#}\left(f_{\beta_{i}, \mu_{i}}^{\#}\right) ; \\
& \Omega^{\mathcal{R}_{i}} \otimes \Omega^{\mathcal{R}_{i}} \rightarrow \tilde{\Omega}^{\mathcal{R}_{i}},
\end{aligned}
$$

where $a_{i}^{\#}$ stands for $a_{i}$ or $a_{i}^{*}$, and $\tilde{\Omega}^{\mathcal{R}_{i}}$ is the vacuum state in $\mathcal{F}^{\mathcal{R}_{i}}\left(L^{2}(\mathbf{R}, \mathcal{B})\right)$. Using Eqs. (27) and (28), one readily verifies that

$$
\begin{aligned}
\left\langle\tilde{\Omega}^{\mathcal{R}_{i}}, a_{i}^{*}\left(f_{\beta_{i}, \mu_{i}}\right) a_{i}\left(f_{\beta_{i}, \mu_{i}}\right) \tilde{\Omega}^{\mathcal{R}_{i}}\right\rangle & =\left\langle\tilde{\Omega}^{\mathcal{R}_{i}}, a_{i}^{*}\left(f_{\beta_{i}, \mu_{i}}^{\#}\right) a_{i}\left(f_{\beta_{i}, \mu_{i}}^{\#}\right) \tilde{\Omega}^{\mathcal{R}_{i}}\right\rangle \\
& =\omega^{\mathcal{R}_{i}}\left(b_{i}^{*}(f) b_{i}(f)\right) \\
& =\left(f, \rho_{\beta_{i}, \mu_{i}}(\cdot) f\right) .
\end{aligned}
$$

Moreover, the free Liouvillean on $\mathcal{F}^{\mathcal{R}_{i}}\left(L^{2}(\mathbf{R} ; \mathcal{B})\right)$ for the reservoir $\mathcal{R}_{i}$ is mapped to

$$
\mathcal{L}^{\mathcal{R}_{i}}=d \Gamma_{i}\left(u_{i}\right)
$$

where $u_{i} \in \mathbf{R}$.

\subsection{The Coupled System}

The kinematical algebra of the total system, $\Sigma \vee \mathcal{R}_{1} \vee \cdots \vee \mathcal{R}_{n}$, is given by

$$
\mathcal{O}=\mathcal{O}^{\Sigma} \otimes \mathcal{O}^{\mathcal{R}_{i}} \otimes \cdots \otimes \mathcal{O}^{\mathcal{R}_{n}},
$$

and the Heisenberg-picture dynamics of the uncoupled system is given by

$$
\alpha_{0}^{t}=\alpha_{\Sigma}^{t} \otimes \alpha_{\mathcal{R}_{i}}^{t} \otimes \cdots \otimes \alpha_{\mathcal{R}_{n}}^{t} .
$$

The representation of $\mathcal{O}$ on $\mathcal{H}:=\mathcal{H}^{\Sigma} \otimes \mathcal{H}^{\Sigma} \otimes \mathcal{F}^{\mathcal{R}_{1}}\left(L^{2}(\mathbf{R} ; \mathcal{B})\right) \otimes \cdots \otimes$ $\mathcal{F}^{\mathcal{R}_{n}}\left(L^{2}(\mathbf{R} ; \mathcal{B})\right)$, determined by the initial state

$$
\omega=\omega^{\Sigma} \otimes \omega^{\mathcal{R}_{1}} \otimes \cdots \otimes \omega^{\mathcal{R}_{n}}
$$

by the GNS construction, is given by

$$
\pi=\pi_{\Sigma} \otimes \pi_{\beta_{1}} \otimes \cdots \otimes \pi_{\beta_{n}},
$$

and an anti-representation commuting with $\pi$ by

$$
\pi^{\#}=\pi_{\Sigma}^{\#} \otimes \pi_{\beta_{1}}^{\#} \otimes \cdots \otimes \pi_{\beta_{n}}^{\#} .
$$

Moreover, let $\Omega: \Omega^{\Sigma} \otimes \tilde{\Omega}_{1}^{\mathcal{R}} \otimes \cdots \otimes \tilde{\Omega}^{\mathcal{R}_{n}}$ denote the vector in $\mathcal{H}$ corresponding to the state $\omega$. Denote the double commutant of $\pi(\mathcal{O})$ by $\mathcal{M}:=\pi(\mathcal{O})^{\prime \prime}$, which is the smallest von Neumann algebra containing $\pi(\mathcal{O})$.

The Liouvillean of the total uncoupled system is given by

$$
\mathcal{L}_{0}=\mathcal{L}^{\Sigma}+\sum_{i=1}^{n} \mathcal{L}^{\mathcal{R}_{i}}
$$


This defines a selfadjoint operator on $\mathcal{H}$.

For $a \in \mathcal{O}$ we abreviate $\pi(a)$ by $a$ whenever there is no danger of confusion. The modular operator of the total system is

$$
\Delta=\Delta^{\Sigma} \otimes \Delta^{\mathcal{R}_{1}} \otimes \cdots \otimes \Delta^{\mathcal{R}_{n}},
$$

and the modular conjugation is

$$
J=J^{\Sigma} \otimes J^{\mathcal{R}_{1}} \otimes \cdots \otimes J^{\mathcal{R}_{n}} .
$$

According to Tomita-Takesaki theory,

$$
J \mathcal{M} J=\mathcal{M}^{\prime}, \Delta^{i t} \mathcal{M} \Delta^{-i t}=\mathcal{M},
$$

for $t \in \mathbf{R}$; (see for example Ref. 7). Furthermore, for $a \in \mathcal{M}$,

$$
J \Delta^{1 / 2} a \Omega=a^{*} \Omega .
$$

The system $\Sigma$ is coupled to the reservoirs $\mathcal{R}_{1}, \ldots, \mathcal{R}_{n}$, through an interaction $g V(t)$, where $V(t) \in \mathcal{O}$ is given by

$$
V(t)=\sum_{i=1}^{n}\left\{\sigma_{-} \otimes b_{i}^{*}\left(f_{i}(t)\right)+\sigma_{+} \otimes b_{i}\left(f_{i}(t)\right)\right\},
$$

where $\sigma_{ \pm}=\sigma_{1} \pm i \sigma_{2}$, and $f_{i}(t) \in L^{2}\left(\mathbf{R}^{+} ; \mathcal{B}\right), i=1, \ldots, n$, are form factors. ${ }^{7}$

The standard Liouvillean of the interacting system acting on the GNS Hilbert space $\mathcal{H}$ is given by

$$
\mathcal{L}_{g}(t)=\mathcal{L}_{0}+g I(t),
$$

where the unperturbed Liouvillean is defined in (40), and the interaction Liouvillean determined by the operator $V(t)$ is given by

$$
\begin{aligned}
I(t)= & \{V(t)-J V(t) J\} \\
= & \sum_{i=1}^{n}\left\{\sigma_{-} \otimes \mathbf{1}^{\Sigma} \otimes a_{i}^{*}\left(f_{i, \beta_{i}, \mu}(t)\right)+\sigma_{+} \otimes \mathbf{1}^{\Sigma} \otimes a_{i}\left(f_{i, \beta_{i}, \mu_{i}}(t)\right)\right. \\
& \left.-i \mathbf{1}^{\Sigma} \otimes \sigma_{-} \otimes(-1)^{N_{i}} a_{i}^{*}\left(f_{i, \beta_{i}, \mu}^{\#}(t)\right)-i \mathbf{1}^{\Sigma} \otimes \sigma_{+} \otimes(-1)^{N_{i}} a_{i}\left(f_{i, \beta_{i}, \mu_{i}}^{\#}(t)\right)\right\},
\end{aligned}
$$

where $a_{i}, a_{i}^{*}$ are the annihilation and creation operators on the fermionic Fock space $\mathcal{F}^{\mathcal{R}_{i}}\left(L^{2}(\mathbf{R} ; \mathcal{B})\right)$. Note that since the perturbation is bounded, the domain of $\mathcal{L}_{g}(t)$ is $\mathcal{D}\left(\mathcal{L}_{g}(t)\right)=\mathcal{D}\left(\mathcal{L}_{0}\right)$.

\footnotetext{
${ }^{7}$ Note that this form of an interaction preserves the total number of fermions in $\Sigma \vee \mathcal{R}_{1} \vee \cdots \vee \mathcal{R}_{n}$, as required by gauge invariance of the first kind.
} 
Let $\bar{U}_{g}$ be the propagator generated by the standard Liouvillean. It satisfies

$$
\partial_{t} \bar{U}_{g}(t)=-i \mathcal{L}_{g}(t) \bar{U}_{g}(t) ; \bar{U}_{g}(0)=1 .
$$

The Heisenberg-picture evolution is given by

$$
\left.\alpha_{g}^{t}(a)=\bar{U}_{g}^{*}(t) a \bar{U}_{g}(t)\right]
$$

for $a \in \mathcal{O}$.

Generally, the kernel of $\mathcal{L}_{g}(t), \operatorname{Ker} \mathcal{L}_{g}$, is expected to be empty when at least two of the reservoirs have different temperatures; (see Sec. 6 and Refs. 16, 20 and 21). ${ }^{8}$ This motivates introducing the so called C-Liouvillean, $L_{g}$, which generates dynamics on a Banach space contained in $\mathcal{H}$ (isomorphic to $\mathcal{O}$ ) and which, by construction, has a non-trivial kernel.

Consider the Banach space

$$
C(\mathcal{O}, \Omega):=\{a \Omega: a \in \mathcal{O}\},
$$

with norm $\|a \Omega\|_{\infty}=\|a\|$. Since $\Omega$ is separating for $\mathcal{O}$, the norm $\|a \Omega\|_{\infty}$ is well-defined, and since $\Omega$ is cyclic for $\mathcal{O}, C(\mathcal{O}, \Omega)$ is dense in $\mathcal{H}$.

We set $\phi(a)=a \Omega$, and define a propagator $U_{g}\left(t, t^{\prime}\right)$ by

$$
\phi\left(\alpha_{g}^{t, t^{\prime}}(a)\right)=U_{g}\left(t, t^{\prime}\right) a \Omega .
$$

Then

$$
\partial_{t} U_{g}\left(t, t^{\prime}\right)=i L_{g}(t) U_{g}\left(t, t^{\prime}\right) \quad \text { with } \quad U_{g}(t, t)=1,
$$

and

$$
U_{g}\left(t^{\prime}, t\right) \Omega=\Omega .
$$

Differentiating (47) with respect to $t$, setting $t=t^{\prime}$, and using (48), (46) and (41), one obtains

$$
\begin{aligned}
{\left[\left(\mathcal{L}_{0}+g V(t)\right) a-a\left(\mathcal{L}_{0}+g V(t)\right)\right] \Omega } & =\left[\left(\mathcal{L}_{0}+g V(t)\right) a-\left(V(t) a^{*}\right)^{*}\right] \Omega \\
& =\left(\mathcal{L}_{0}+g V(t)-g J \Delta^{1 / 2} V(t) \Delta^{-1 / 2} J\right) a \Omega \\
& \equiv L_{g}(t) a \Omega .
\end{aligned}
$$

Hence, the C-Liouvillean is given by

$$
L_{g}(t):=\mathcal{L}_{0}+g V(t)-g J \Delta^{1 / 2} V(t) \Delta^{-1 / 2} J .
$$

\footnotetext{
${ }^{8}$ This is consistent with the fact that the coupled system is not expected to possess the property of return to equilibrium if the reservoirs have different temperatures (or chemical potentials). One can verify that, indeed, this is the case when assumptions (A1)-(A3), below, are satisfied.
} 
Note that, by construction,

$$
L_{g}(t) \Omega=0
$$

for all $t \in \mathbf{R}$.

Next, we discuss the assumptions on the interaction. For $\delta>0$, we define the strips in the complex plane

$$
I(\delta):=\{z \in \mathbf{C}:|\operatorname{Imz}|<\delta\}
$$

and

$$
I^{-}(\delta):=\{z \in \mathbf{C}:-\delta<\operatorname{Im} z<0\} .
$$

Moreover, for every function $f \in L^{2}\left(\mathbf{R}^{+} ; \mathcal{B}\right)$, we define a function $\tilde{f}$ by setting

$$
\tilde{f}:=\left\{\begin{array}{ll}
\sqrt{m(u)}(u, \sigma), & u \geq 0 \\
\sqrt{m(|u|)} \bar{f}(|u|, \sigma), & u \geq 0
\end{array},\right.
$$

where $m(u) d u$ is the measure on $\mathbf{R}^{+}$. Denote by $H^{2}(\delta, \mathcal{B})$ the Hardy class of analytic functions

$$
h: I(\delta) \rightarrow \mathcal{B}
$$

with

$$
\|h\|_{H^{2}(\delta, \mathcal{B})}:=\sup _{|\theta|<\delta} \int_{\mathbf{R}}\|h(u+i \theta)\|_{\mathcal{B}}^{2} d u<\infty .
$$

We require the following basic assumptions on the interaction term.

(A1) Periodicity.

The interaction term $V(t)$ is periodic with (a minimal) period $\tau<$ $\infty: V(t)=V(t+\tau)$.

(A2) Regularity of the form factors.

Assume that $\exists \delta>0$, independent of $t$ and $i \in\{1, \ldots, n\}$, such that

$$
e^{-\beta_{i} u / 2} f_{\beta_{i}, u_{i}}(u, t) \in H^{2}(\delta, \mathcal{B}),
$$

where $f_{i}$ is as in Eq. (42) and $f_{\beta, \mu}$ is defined in (29).

(A3) Fermi Golden Rule.

We assume that

$$
\sum_{i=1}^{n}\left\|\tilde{f}_{i}\left(2 \omega_{0}, t\right)\right\|_{\mathcal{B}}^{2}>0
$$


where $\tilde{f}_{i}$, is defined in (52). which means that the coupling of $\Sigma$ to the reservoirs is non-vanishing in second order perturbation theory. ${ }^{9}$

Let $\hat{f}$ be the Fourier transform of $\tilde{f}$ given by

$$
\hat{f}_{m}(u, \omega)=\frac{1}{\tau} \int_{0}^{\tau} d t e^{-i m \omega t} \tilde{f}(u, t),
$$

where $\omega=\frac{2 \pi}{\tau}$, and $\tau$ is the period of the interaction term (see assumption (A1)). Then $\tilde{f}(u, t)=\sum_{m \in \mathbf{Z}} e^{i m \omega t} \hat{f}_{m}(u, \omega)$. It follows from (A2) and Parseval's theorem that

$$
\sum_{m \in \mathbf{Z}}\left\|\hat{f}_{m}(u+m \omega, \omega)\right\|_{\mathcal{B}}^{2}<\infty
$$

for $u \in \mathbf{R}$.

Let $\tilde{U}_{g}$ be the propagator generated by the adjoint of the C-Liouvillean, ie,

$$
\begin{aligned}
\partial_{t} \tilde{U}_{g}\left(t, t^{\prime}\right) & =-i \tilde{U}_{g}\left(t, t^{\prime}\right) L_{g}^{*}(t), \\
\tilde{U}_{g}(t, t) & =1 .
\end{aligned}
$$

Assumption (A2) implies that the perturbation is bounded, and hence the domain of $L_{g}^{\#}$, where $L_{g}^{\#}$ stands for $L_{g}$ or $L_{g}^{*}$, is

$$
\mathcal{D}\left(L_{g}^{\#}\right)=\mathcal{D}\left(\mathcal{L}_{0}\right),
$$

and $U_{g}, \tilde{U}_{g}$ are bounded and strongly continuous in $t$ and $t^{\prime}$.

\section{THE FLOQUET LIOUVILLEAN}

In this section, we extend Floquet theory for periodically driven quantum systems at zero temperature to a theory for systems at positive temperatures. The goal is to investigate whether the state of the coupled system converges to a time-periodic state. We introduce the Floquet Liouvillean, which generates the dynamics on a suitable Banach space, and we show in the following section that the time-periodic state to which the state of the system converges after very many periods is related to a zero-energy resonance of the Floquet Liouvillean.

We consider the extended Hilbert space $\tilde{\mathcal{H}}:=L^{2}([0, \tau]) \otimes \mathcal{H}$, where $\tau$ is the period of the perturbation appearing in (42) and (A1), and we introduce the

\footnotetext{
${ }^{9}$ For instance, when the reservoirs are formed of nonrelativistic fermions in $\mathbf{R}^{3}$, an example of a form factor satisfying assumptions (A1)-(A3) is given by

$$
f_{i}(u, t)=h_{i}(t)|u|^{n} e^{-|u|^{2}}
$$

where $h_{i}(t)$ is a bounded, periodic function of $\mathrm{t} \in \mathbf{R}$ and $n \geq 2$.
} 
Floquet Liouvillean

$$
K_{g}^{*}:-i \partial_{t} \otimes \mathbf{1}+\mathbf{1} \otimes L_{g}^{*}(t),
$$

with periodic boundary conditions in $t$. Note that, under assumption (A2), $K_{g}^{*}$ is a closed operator with domain $\mathcal{D}=\mathcal{D}\left(i \partial_{t} \otimes \mathbf{1}\right) \cap \mathcal{D}\left(\mathbf{1} \otimes \mathcal{L}_{0}\right)$.

By Fourier transformation, $\tilde{\mathcal{H}}$ is isomorphic to

$$
\bigoplus_{n \in \mathbf{Z}}\left\langle e^{i n \omega t}\right\rangle \otimes \mathcal{H}=\bigoplus_{n \in \mathbf{Z}} \mathbf{h}^{(n)} \otimes \mathcal{H},
$$

where $\mathbf{h}^{(n)}:=\left\langle e^{i n \omega t}\right\rangle$ and $\omega=\frac{2 \pi}{\tau}$.

According to Floquet theory of quantum mechanical systems driven by periodic perturbation, $(11,25,26)$, the semi-group generated by $K_{g}^{*}$ is given by

$$
\left(e^{-i \sigma K_{g}^{*}} f\right)(t)=\tilde{U}_{g}(t, t-\sigma) f(t-\sigma),
$$

where $f \in \tilde{\mathcal{H}}$ and $\sigma \in \mathbf{R}$. Relation (58) can be seen by differentiating both sides with respect to $\sigma$ and setting $\sigma=0$ (see Ref. 11). (Alternatively, one may use the Trotter product formula, (23).)

Note that if

$$
K_{g}^{*} \phi=\lambda \phi
$$

for $\phi \equiv \phi(t) \in \tilde{\mathcal{H}}$ and $\lambda \in \mathbf{C}$, then $\phi(t)$ satisfies

$$
\tilde{U}_{g}(t, 0) \phi(0)=e^{-i \lambda t} \phi(t) \text {. }
$$

Conversely, if

$$
\tilde{U}_{g}(\tau, 0) \phi(0)=e^{-i \lambda \tau} \phi_{0}
$$

then

$$
\phi(t)=e^{i \lambda t} \tilde{U}_{g}(t, 0) \phi_{0}
$$

is an eigenfunction of $K_{g}^{*}$ with eigenvalue $\lambda$.

We now study the spectrum of $K_{g}^{*}$ using complex spectral deformation techniques as developed in Refs. $12,14-16 .^{10}$

Let $\mathbf{u}_{i}$ be the unitary transformation generating translations in energy for the $i$ th reservoir, $i=1, \ldots, n$. More precisely, for $f_{i} \in L^{2}(\mathbf{R} ; \mathcal{B})$,

$$
\mathbf{u}_{i}(\theta) f_{i}(u)=f_{i}^{\theta}(u)=f_{i}(u+\theta) .
$$

Moreover, let

$$
U_{i}(\theta):=\Gamma_{i}\left(\mathbf{u}_{i}(\theta)\right)
$$

denote the second quantization of $\mathbf{u}_{i}(\theta)$.

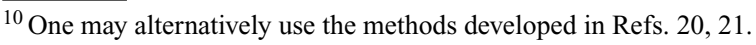


Explicitly, $U_{i}(\theta)=e^{i \theta A_{i}}$, where $A_{i}:=i d \Gamma_{i}\left(\partial_{u_{i}}\right)$ is the second quantization of the generator of energy translations for the $i$ th reservoir, $i=1, \ldots, n$. We set

$$
U(\theta):=\mathbf{1}^{\Sigma} \otimes \mathbf{1}^{\Sigma} \otimes U_{1}(\theta) \otimes \cdots \otimes U_{n}(\theta) .
$$

Let

$$
\begin{aligned}
K_{g}^{*}(\theta) & :=U(\theta) K_{g}^{*} U(-\theta) \\
& =-i \partial_{t}+L_{g}^{*}(t, \theta),
\end{aligned}
$$

where $L_{g}^{*}(t, \theta)$ is given by

$$
\begin{gathered}
L_{g}^{*}(t, \theta):=U(\theta) L_{g}^{*}(t) U(-\theta)=\mathcal{L}_{0}+N \theta+g \tilde{V}^{\mathrm{tot}}(t, \theta), \\
\mathcal{L}_{0}=\mathcal{L}^{\Sigma}+\sum_{i} \mathcal{L}^{\mathcal{R}_{i}}, \mathcal{L}^{\mathcal{R}_{i}}=d \Gamma\left(u_{i}\right), i=1, \ldots, n, \text { and } \\
\tilde{V}^{\mathrm{tot}}(t, \theta)=\sum_{i}\left\{\sigma_{+} \otimes \mathbf{1}^{\Sigma} \otimes a_{i}\left(f_{i, \beta_{i}, \mu_{i}}^{(\theta)}(t)\right)+\sigma_{-} \otimes \mathbf{1}^{\Sigma} \otimes a_{i}^{*}\left(f_{i, \beta_{i}, \mu_{i}}^{\theta}(t)\right)\right. \\
-i \mathbf{1}^{\Sigma} \otimes \sigma_{-} \otimes(-1)^{N_{i}}\left(a_{i}\left(e^{\beta_{i}\left(u_{i}-\mu_{i}\right) / 2} f_{i, \beta_{i}, \mu_{i}}^{\#(\theta)}(t)\right)\right. \\
-i \mathbf{1}^{\Sigma} \otimes \sigma_{+} \otimes(-1)^{N_{i}} a_{i}^{*}\left(e^{-\beta_{i}\left(u_{i}-\mu_{i}\right) / 2} f_{i, \beta_{i}, \mu_{i}}^{\#(\theta)} .\right.
\end{gathered}
$$

It follows from assuption (A2) that, for $\theta \in I(\delta), \tilde{V}_{g}^{\text {tot }}(t, \theta)$ is a bounded operator. Hence $K_{g}^{*}(t, \theta)$ is well-defined and closed on the domain $\mathcal{D}:=\mathcal{D}\left(i \partial_{t}\right) \cap$ $\mathcal{D}(N) \cap \mathcal{D}\left(\mathcal{L}^{\mathcal{R}_{1}}\right) \cap \cdots \cap \mathcal{D}\left(\mathcal{L}^{\mathcal{R}_{n}}\right)$. When the coupling $g=0$, the pure point spectrum of $\mathcal{L}_{0}$ is $\sigma_{p p}\left(\mathcal{L}_{0}\right)=\left\{-2 \omega_{0}, 0,2 \omega_{0}\right\}$, with double degeneracy at 0 , and the continuous spectrum of $\mathcal{L}_{0}$ is $\sigma_{\text {cont }}\left(\mathcal{L}_{0}\right)=\mathbf{R}$. It follows that

$$
\sigma_{p p}\left(K_{0}\right)=\left\{E_{j}^{(k)}(g=0)=E_{j}+k \omega: j=0, \ldots, 3, k \in \mathbf{Z}\right\},
$$

where $E_{0,1}=0, E_{2}=-2 \omega_{0}$, and $E_{3}=2 \omega_{0}$, and $\sigma_{c o n t}\left(K_{0}\right)=\mathbf{R}$. Let

$$
K^{\Sigma}:=-i \partial_{t}+\mathcal{L}^{\Sigma} .
$$

Clearly, $\sigma\left(K^{\Sigma}\right)=\sigma_{p p}\left(K_{0}\right)$. We have the following two easy lemmas.

Lemma 4.1. For $\theta \in \boldsymbol{C}$, the following holds.

(i) For any $\psi \in \mathcal{D}$, one has

$$
\left\|K_{0}(\theta) \psi\right\|^{2}=\left\|K_{0}(\operatorname{Re} \theta) \psi\right\|^{2}+|\operatorname{Im} \theta|^{2}\|N \psi\|^{2} .
$$

(ii) If $\operatorname{Im} \theta \neq 0$, then $K_{0}(\theta)$ is a normal operator satisfying

$$
K_{0}(\theta)^{*}=K_{0}(\bar{\theta}),
$$

and $\mathcal{D}\left(K_{0}(\theta)\right)=\mathcal{D}$. 
(iii) The spectrum of $K_{0}(\theta)$ is

$$
\begin{gathered}
\sigma_{\text {cont }}\left(K_{0}(\theta)\right)=\{n \theta+s: n \in N \backslash\{0\} \text { and } s \in \boldsymbol{R}\}, \\
\sigma_{p p}\left(K_{0}(\theta)\right)=\left\{k \omega+E_{j}: j=0, \ldots, 3, k \in \boldsymbol{Z}\right\},
\end{gathered}
$$

where $E_{0,1}=0, E_{2}=-2 \omega_{0}$ and $E_{3}=2 \omega_{0}$, (the eignevaues of $\left.\mathcal{L}^{\Sigma}\right)$, and $\omega=\frac{2 \pi}{\tau}$.

Proof: The first claim follows directly by looking at the sector where $N=n \mathbf{1}$, since $K_{0}(\theta)$ restricted to this sector is reduced to

$$
K_{0}^{(n)}(\theta)=K^{\Sigma}+s_{1} \cdots+s_{n}+n \theta,
$$

where $s_{1}, \ldots, s_{n}$ are interpreted as one-particle multiplication operators. For $\operatorname{Im} \theta \neq 0$, it also follows from (72) that

$$
\mathcal{D}=\left\{\psi=\left\{\psi^{(n)}\right\}: \psi^{(n)} \in \mathcal{D}\left(K_{0}^{(n)}(\theta)\right) \quad \text { and } \quad \sum_{n}\left\|K_{0}^{(n)}(\theta) \psi^{(n)}\right\|^{2}<\infty\right\},
$$

and hence $K_{0}(\theta)$ is a closed normal operator on $\mathcal{D}$. Claims (ii) and (iii) follow from the corresponding statements on $K_{0}^{(n)}(\theta)$.

Lemma 4.2. Suppose (A1)-(A3) hold, and assume that $(g, \theta) \in \boldsymbol{C} \times I^{-}(\delta)$. Then the following holds.

(i) $\mathcal{D}\left(K_{g}^{*}(\theta)\right)=\mathcal{D}$ and $\left(K_{g}^{*}(\theta)\right)^{*}=K_{\bar{g}}(\bar{\theta})$.

(ii) The map $(g, \theta) \rightarrow K_{g}^{*}(\theta)$ from $\boldsymbol{C} \times I^{-}(\delta)$ to the set of closed operators on $\tilde{\mathcal{H}}$ is an analytic family (of type $A$ ) in each variable separately; (see Ref. 18, chapter V, Sec. 3.2).

(iii) For finite $g \in \mathbf{R}$ and Imz large enough,

$$
s-\lim _{\operatorname{Im} \theta \uparrow 0}\left(K_{g}^{*}(\theta)-z\right)^{-1}=\left(K_{g}^{*}(\operatorname{Re} \theta)-z\right)^{-1} .
$$

Proof: The first claim (i) follows from the fact that $g \tilde{V}^{\text {tot }}(t, \theta)$ is bounded for $\theta \in I(\delta)$. It also follows from assumption (A2) that $(g, \theta) \rightarrow K_{g}^{*}(\theta)$ is analytic in $\theta \in I^{-}(\delta)$. Analyticity in $g$ is obvious from (65). We still need to prove claim (iii). Without loss of generality, assume that $\operatorname{Re} \theta=0$. It follows from assumption (A2) that the resolvent formula

$$
\left(K_{g}^{*}(\theta)-z\right)^{-1}=\left(K_{0}^{*}(\theta)-z\right)^{-1}\left(1+g \tilde{V}^{\text {tot }}(\cdot, \theta)\left(K_{0}^{*}(\theta)-z\right)^{-1}\right)^{-1},
$$

holds for small $g$, as long as $z$ belongs to the half-plane $\{z \in \mathbf{C}: 0<c<\operatorname{Imz}\}$. Since $\left(K_{0}^{*}(\theta)-z\right)^{-1}$ is uniformly bounded as $\operatorname{Im} \theta \uparrow 0$ for $g \in \mathbf{R}$ finite and $\operatorname{Im} z$ large enough, and $\tilde{V}^{\text {tot }}(\theta)$ is bounded and analytic in $\theta$, claim (iii) follows from the Neumann series expansion of the resolvent of $K_{g}^{*}(\theta)$. 
Next, we apply degenerate perturbation theory, as developed in Ref. 12, to compute the spectrum of $K_{g}^{*}(\theta)$. Using contour integration, one may define the projection onto the perturbed eigenstates of $K_{g}^{*}(\theta)$, for $\operatorname{Im} \theta \in I^{-}(\delta)$. Let

$$
\tilde{P}_{g,(k)}(\theta):=\oint_{\gamma k} \frac{d z}{2 \pi i}\left(z-K_{g}^{*}(\theta)\right)^{-1},
$$

where $\gamma_{k}$ is a contour that encloses the eigenvalues $E_{j}^{(k)}(g=0), j=0, \ldots, 3, k \in$ $\mathbf{Z}$, at a distance $d>0$, such that, for sufficiently small $|g|$ (to be specified below) the contour also encloses $E_{j}^{(k)}(g)$.

Moreover, let $\tilde{T}_{g,(k)}:=\tilde{P}_{0,(k)} \tilde{P}_{g,(k)}(\theta) \tilde{P}_{0(k) \cdot}{ }^{11}$ We show in Theorem 4.3 that the isomorphism

$$
\tilde{S}_{g,(k)}(\theta):=\tilde{T}_{g,(k)}^{-1 / 2} \tilde{P}_{0,(k)} \tilde{P}_{g,(k)}(\theta): \operatorname{Ran}\left(\tilde{P}_{g, k}(\theta)\right) \rightarrow \mathbf{h}^{(k)} \otimes \mathcal{H}^{\Sigma} \otimes \mathcal{H}^{\Sigma}
$$

has an inverse

$$
\tilde{S}_{g,(k)}^{-1}(\theta):=\tilde{P}_{g,(k)}(\theta) \tilde{P}_{0,(k)} \tilde{T}_{g,(k)}^{-1 / 2}(t): \mathbf{h}^{(k)} \otimes \mathcal{H}^{\Sigma} \otimes \mathcal{H}^{\Sigma} \rightarrow \operatorname{Ran}\left(\tilde{P}_{g,(k)}(\theta)\right) .
$$

We set

$$
\tilde{M}_{g,(k)}:=\tilde{P}_{0,(k)} \tilde{P}_{g,(k)}(\theta) K_{g}^{*}(\theta) \tilde{P}_{g,(k)}(\theta) \tilde{P}_{0,(k)},
$$

and define the quasi-Floquet Liouvillean by

$$
\tilde{\Sigma}_{g,(k)}:=\tilde{S}_{g,(k)}(\theta) \tilde{P}_{g,(k)}(\theta) K_{g}^{*}(\theta) \tilde{P}_{g,(k)}(\theta) \tilde{S}_{g,(k)}^{-1}(\theta)=\tilde{T}_{g,(k)}^{-1 / 2} \tilde{M}_{g,(k)} \tilde{T}_{g,(k)}^{-1 / 2} .
$$

Let $\kappa=\min \left\{\delta, \frac{\pi}{\beta_{1}}, \ldots, \frac{\pi}{\beta_{n}}\right\}$, where $\delta$ appears in assumption (A2), Sec. 3, and $\beta_{1}, \ldots, \beta_{n}$, are the inverse temperatures of the reservoirs $\mathcal{R}_{1}, \ldots, \mathcal{R}_{n}$, respectively. For $\theta \in I^{-}(\kappa)($ see $(51))$, we choose a parameter $v$ such that

$$
-\kappa<\nu<0 \text { and }-\kappa<\operatorname{Im} \theta<-\frac{\kappa+|\nu|}{2} \text {. }
$$

We also choose a constant $g_{1}>0$ such that

$$
g_{1} C<(\kappa-|\nu|) / 2
$$

where

$$
C:=\sup _{\theta \in I(\delta), t \in \mathbf{R}}\left\|\tilde{V}^{\text {tot }}(t, \theta)\right\|<\infty
$$

Theorem 4.3. Suppose that assumptions (A1)-(A3) hold. Then, for $g_{1}>0$ satisfying (81), $\theta \in I^{-}(k)$ and $v$ satisfying (80), the following holds.

\footnotetext{
${ }^{11}$ Note that, although $K_{0}^{*}(\theta)$ is unbounded, it is a normal operator, and hence $\tilde{P}_{0,(k)}$ is well-defined by the spectral theorem: (see for example Ref.18).
} 
(i) If $|g|<g_{1}$, the essential spectrum of the operator $K_{g}^{*}(\theta)$ is contained in the half-plane $\mathbf{C} \backslash \Xi(v)$, where $\Xi(v):=\{z \in \mathbf{C}:$ Im $z \geq v\}$. Moreover, the discrete spectrum of $K_{g}^{*}(\theta)$ is indepenent of $\theta \in I^{-}(K)$. If $|g|<1 / 2 g_{1}$, then the spectral projections $\tilde{P}_{g,(k)}(\theta), k \in \mathbf{Z}$, associated to the spectrum of $K_{g}^{*}(\theta)$ in the half-plane $\Xi(v)$, are analytic in $\mathrm{g}$ and satisfy the estimate

$$
\left\|\tilde{P}_{g,(k)}(\theta)-\tilde{P}_{0,(k)}\right\|<1 .
$$

(ii) If $|g|<\frac{g_{1}}{2}$, then the quasi-Floquet Liouvillean $\tilde{\Sigma}_{g,(k)}$ defined in (79) depends analytically on $g$, and has a Taylor expansion

$$
\tilde{\Sigma}_{g,(k)}=K_{(k)}^{\Sigma}+\sum_{j=1}^{\infty} g^{2 j} \tilde{\Sigma}_{(k)}^{(2 j)}
$$

where

$$
K_{(k)}^{\Sigma}:=k \omega+\mathcal{L}^{\Sigma}, k \in \boldsymbol{Z}
$$

The first non-trivial coefficient in (84) is

$$
\tilde{\Sigma}_{(k)}^{(2)}=\frac{1}{2} \oint_{\gamma_{k}} \frac{d z}{2 \pi i}\left(\xi_{(k)}(z)\left(z-K_{(k)}^{\Sigma}\right)^{-1}+\left(z-K_{(k)}^{\Sigma}\right)^{-1} \xi_{(k)}(z)\right),
$$

where $\xi_{(k)}(z)=\tilde{P}_{0,(k)} \tilde{V}^{\text {tot }}(\theta)\left(z-K_{0}(\theta)\right)^{-1} \tilde{V}^{\text {tot }}(\theta) \tilde{P}_{0,(k)}$.

Proof: (i) The resolvent formula

$$
\left(K_{g}^{*}(\theta)-z\right)^{-1}=\left(K_{0}^{*}(\theta)-z\right)^{-1}\left(1+g \tilde{V}^{\text {tot }}(\cdot, \theta)\left(K_{0}^{*}(\theta)-z\right)^{-1}\right)^{-1},
$$

holds for small $g$ and $z$ in the half-plane $\{z \in \mathbf{C}: 0<c<\operatorname{Im} z\}$. We extend the domain of validity of (86) by refining the estimate on $g \tilde{V}^{\text {tot }}(t, \theta)\left(K_{0}^{*}(\theta)-z\right)^{-1}$.

Note that

$$
\begin{aligned}
\left\|g \tilde{V}^{\text {tot }}(\cdot, \theta)\left(K_{0}^{*}(\theta)-z\right)^{-1}\right\| & \leq|g| C\left\|\left(K_{0}(\theta)-z\right)^{-1}\right\| \\
& \leq|g| C \frac{1}{\operatorname{dist}\left(z, \eta\left(K_{0}^{*}(\theta)\right)\right)},
\end{aligned}
$$

where $C$ is given by $(82)$ and $\eta\left(K_{0}^{*}(\theta)\right)$ is the closure of the numerical range of $K_{0}^{*}$. Fix $g_{1}$ such that it satisfies $(81)$, and choose $\epsilon$ such that $\epsilon>\frac{k-|v|}{2}>0$. Let

$$
G(v, \epsilon):=\left\{z \in \mathbf{C}: \operatorname{Imz}>v ; \operatorname{dist}\left(z, \eta\left(K_{0}^{*}(\theta)\right)\right)>\epsilon\right\} .
$$

Then

$$
\sup _{z \in G(\nu, \epsilon)}\left\|g \tilde{V}^{\mathrm{tot}}(\cdot, \theta)\left(K_{0}^{*}(\theta)-z\right)^{-1}\right\| \leq \frac{|g|}{g_{1}} .
$$


If $|g|<g_{1}$, the resolvent formula (86) holds on $G(\nu, \epsilon)$, and, for $m \geq 1$,

$$
\sup _{z \in G(v, \epsilon)}\left\|\left(z-K_{g}^{*}(\theta)\right)^{-1}-\sum_{j=0}^{m-1}\left(z-K_{0}^{*}(\theta)\right)^{-1}\left(g \tilde{V}^{\text {tot }}(\cdot, \theta)\left(z-K_{0}^{*}(\theta)\right)^{-1}\right)^{j}\right\| \leq \frac{\left(\frac{|g|}{g_{1}}\right)^{m}}{1-\frac{|g|}{g_{1}}} .
$$

It follows that

$$
\bigcup_{\epsilon>\frac{\kappa-|\nu|}{2}} G(\nu, \epsilon) \subset \rho\left(K_{g}^{*}(\theta)\right),
$$

where $\rho\left(K_{g}^{*}(\theta)\right)$ is the resolvent set of $K_{g}^{*}(\theta)$. Moreover, setting $m=1$ in (87), it follows that, for $|g|<g_{1} / 2$,

$$
\left\|\tilde{P}_{g,(k)}(\theta)-\tilde{P}_{0,(k)}\right\|<1,
$$

and hence $\tilde{P}_{g,(k)}$ is analytic in $g$. We still need to prove the independence of $\sigma_{p p}\left(K_{g}^{*}(\theta)\right)$ of $\theta \in I^{-}(\kappa)$.

Fix $\left(g_{0}, \theta_{0}\right) \in \mathbf{C} \times I^{-}(\delta)$ such that $\left|g_{0}\right|<g_{1}$. The discrete eigenvalues of $K_{g_{0}}^{*}(\theta)$ are analytic functions with at most algebraic singularities in the neighbourhood of $\theta_{0}$, since $K_{g_{0}}^{*}(\theta)$ is analytic in $\theta$. Moreover, since $K_{g_{0}}^{*}\left(\theta_{0}\right)$ and $K_{g_{0}}^{*}(\theta)$ are unitarily equivalent if $\left(\theta-\theta_{0}\right) \in \mathbf{R}$, it follows that the pure point spectrum of $K_{g_{0}}^{*}(\theta)$ is independent of $\theta$.

(ii) Analyticity of $\tilde{T}_{g,(k)}$ directly follows from (i) and the definition of $\tilde{T}_{g,(k)}$. Since $\left\|\tilde{T}_{g,(k)}-1\right\|<1$ for $|g|<g_{1} / 2, \tilde{T}_{g,(k)}^{-1 / 2}$ is also analytic in $g$. Inserting the Neumann series for the resolvent of $K_{g}^{*}(\theta)$, gives

$$
\tilde{T}_{g,(k)}=1+\sum_{j=1}^{\infty} g^{j} \tilde{T}_{(k)}^{(j)}
$$

with

$\tilde{T}_{(k)}^{(j)}=\oint_{\gamma k} \frac{d z}{2 \pi i}\left(z-K^{\Sigma}\right)^{-1} \tilde{P}_{0,(k)} \tilde{V}^{\mathrm{tot}}(\cdot, \theta)\left(\left(z-K_{0}^{*}(\theta)\right)^{-1} \tilde{V}^{\mathrm{tot}}(\cdot, \theta)\right)^{j-1} \tilde{P}_{0,(k)}\left(z-K^{\Sigma}\right)^{-1}$.

Similarly,

$$
\tilde{M}_{g,(k)}=K^{\Sigma}+\sum_{j=1}^{\infty} g^{j} \tilde{M}_{(k)}^{(j)}
$$

with

$$
\tilde{M}_{(k)}^{(j)}=\oint_{\gamma_{k}} \frac{d z}{2 \pi i} z\left(z-K^{\Sigma}\right)^{-1} \tilde{P}_{0,(k)} \tilde{V}^{\text {tot }}(\cdot, \theta)\left(\left(z-K_{0}^{*}(\theta)\right)^{-1} \tilde{V}^{\text {tot }}(\cdot, \theta)\right)^{j-1} \tilde{P}_{0,(k)}\left(z-K^{\Sigma}\right)^{-1} .
$$


The odd terms in the above two expansions are zero due to the fact that $\tilde{P}_{0,(k)}$ projects onto the $N=0$ sector. The first non-trivial coefficient in the Taylor series of $\tilde{\Sigma}_{g(k)}$ is

$$
\begin{aligned}
\tilde{\Sigma}_{(k)}^{(2)} & =\tilde{M}_{(k)}^{(2)}-\frac{1}{2}\left(\tilde{T}_{(k)}^{(2)} K^{\Sigma}+K^{\Sigma} \tilde{T}_{(k)}^{(2)}\right) \\
& =\frac{1}{2} \oint_{\gamma k} \frac{d z}{2 \pi i}\left(\xi_{k}(z)\left(z-K^{\Sigma}\right)^{-1}+\left(z-K^{\Sigma}\right)^{-1} \xi_{k}(z)\right),
\end{aligned}
$$

with

$$
\xi_{k}(z)=\tilde{P}_{0(k)} \tilde{V}_{g}^{\text {tot }}(\cdot, \theta)\left(z-K_{0}^{*}(\theta)\right)^{-1} \tilde{V}_{g}^{\text {tot }}(\cdot, \theta) \tilde{P}_{0(k)}
$$

We explicitly compute the discrete spectrum of $K_{g}^{*}(\theta)$ to second order in the coupling constant. Let $e_{1,2} \in \mathcal{H}^{\Sigma}$ denote the vectors of spin up and down respectively. Then the states in $\tilde{\mathcal{H}}$ corresponding to the eigenvalues $E_{j}^{(k)}(g=$ $0), j=0, \ldots, 3, k \in \mathbf{Z}$, are

$$
\begin{aligned}
& \phi_{k}^{0}=e^{i k \omega} \otimes e_{1} \otimes e_{1} \otimes \tilde{\Omega}^{\mathcal{R}_{1}} \otimes \cdots \otimes \tilde{\Omega}^{\mathcal{R}_{n}}, \\
& \phi_{k}^{1}=e^{i k \omega} \otimes e_{2} \otimes e_{2} \otimes \tilde{\Omega}^{\mathcal{R}_{1}} \otimes \cdots \otimes \tilde{\Omega}^{\mathcal{R}_{n}}, \\
& \phi_{k}^{2}=e^{i k \omega} \otimes e_{2} \otimes e_{1} \otimes \tilde{\Omega}^{\mathcal{R}_{1}} \otimes \cdots \otimes \tilde{\Omega}^{\mathcal{R}_{n}}, \\
& \phi_{k}^{3}=e^{i k \omega} \otimes e_{1} \otimes e_{2} \otimes \tilde{\Omega}^{\mathcal{R}_{1}} \otimes \cdots \otimes \tilde{\Omega}^{\mathcal{R}_{n}},
\end{aligned}
$$

where $\Omega^{\mathcal{R}_{i}}$ is the vacuum state in $\mathcal{F}^{\mathcal{R}_{i}}\left(L^{2}(\mathbf{R} ; \mathcal{B})\right)$.

We apply perturbation theory to calculate $E_{j}^{(k)}(g)$. We know that

$$
\begin{aligned}
\tilde{\Sigma}_{(k)}^{(2)}= & \frac{1}{2} \oint_{\gamma k} \frac{d z}{2 \pi i}\left\{\tilde{P}_{0,(k)} \tilde{V}^{\text {tot }}(\cdot, \theta)\left(z-K_{0}(\theta)\right)^{-1} \tilde{V}^{\text {tot }}(\cdot, \theta) \tilde{P}_{0,(k)}\left(z-K_{(k)}^{\Sigma}\right)^{-1}\right. \\
& \left.+\left(z-K_{(k)}^{\Sigma}\right) \tilde{P}_{0,(k)} \tilde{V}^{\text {tot }}(\cdot, \theta)\left(z-K_{0}(\theta)\right)^{-1} \tilde{V}^{\text {tot }}(\cdot, \theta) \tilde{P}_{0,(k)}\right\}
\end{aligned}
$$

For $f_{\beta, \mu}$ as in (29), we let its Fourier transform be

$$
\hat{f}_{\beta, \mu, m}(u, \omega):=\frac{1}{\tau} \int_{0}^{\tau} d t e^{-i m \omega t} f_{\beta, \mu}(u, t) .
$$

Similarly, for $f_{\beta, \mu}^{\#}$ as in (30), we let

$$
\hat{f}_{\beta, \mu, m}^{\#}(u, \omega):=\frac{1}{\tau} \int_{0}^{\tau} d t e^{-i m \omega t} f_{\beta, \mu}^{\#}(u, t) .
$$


Consider first the nondegenerate eigenvalue $E_{3}^{(k)}$. Applying the Cauchy integration formula to (95), and using the facts that

$$
\lim _{\epsilon \searrow 0} \operatorname{Re} \frac{1}{x-i \epsilon}=\mathcal{P} V \frac{1}{x}, \text { and } \lim _{\epsilon \searrow 0} \operatorname{Im} \frac{1}{x-i \epsilon}=\pi \delta(x),
$$

where $\mathcal{P} V$ denotes the Cauchy principal value, it follows that

$$
\begin{aligned}
& R e \ll \phi_{k}^{3}, \tilde{\Sigma}_{3}^{k(2)} \phi_{k}^{3} \gg=\sum_{m \in \mathbf{Z}} \sum_{i=1}^{n} \mathcal{P} V \int_{\mathbf{R}} d u \frac{\left\|\hat{f}_{\beta_{i}, \mu_{i}, m(u, w)}\right\|_{\mathcal{B}}^{2}}{2 \omega_{0}-(k-m) \omega-u}, \\
& I m \ll \phi_{k}^{3}, \tilde{\Sigma}_{3}^{k(2)} \phi_{k}^{3} \gg=-\pi \sum_{m \in \mathbf{Z}} \sum_{i=1}^{n}\left\|\hat{f}_{\beta_{i}, \mu_{i}, m}\left(2 \omega_{0}-(k-m) \omega, \omega\right)\right\|_{\mathcal{B}}^{2},
\end{aligned}
$$

where $\ll \cdot, \gg$ is the scalar product on $\tilde{\mathcal{H}}$. Therefore,

$$
\begin{aligned}
E_{3}^{(k)}(g) & =k \omega+2 \omega_{0}+g^{2} \sum_{m \in \mathbf{Z}} \sum_{i=1}^{n} \mathcal{P} V \int_{\mathbf{R}} d u \frac{\left\|\hat{f}_{\beta_{i}, \mu_{i}, m(u, w)}\right\|_{\mathcal{B}}^{2}}{2 \omega_{0}-(k-m) \omega-u} \\
& -i \pi g^{2} \sum_{m \in \mathbf{Z}} \sum_{i=1}^{n}\left\|\hat{f}_{\beta_{i}, \mu_{i}, m}\left(2 \omega_{0}-(k-m) \omega, \omega\right)\right\|_{\mathcal{B}}^{2}+\mathcal{O}\left(g^{4}\right) .
\end{aligned}
$$

Similarly,

$$
\begin{aligned}
E_{2}^{(k)}(g) & =k \omega-2 \omega_{0}-g^{2} \sum_{m \in \mathbf{Z}} \sum_{i=1}^{n} \mathcal{P} V \int_{\mathbf{R}} d u \frac{\left\|\hat{f}_{\beta_{i}, \mu_{i}, m(u, w)}\right\|_{\mathcal{B}}^{2}}{2 \omega_{0}-(k-m) \omega-u} \\
& -i \pi g^{2} \sum_{m \in \mathbf{Z}} \sum_{i=1}^{n}\left\|\hat{f}_{\beta_{i}, \mu_{i}, m}\left(2 \omega_{0}-(k-m) \omega, \omega\right)\right\|_{\mathcal{B}}^{2}+\mathcal{O}\left(g^{4}\right) .
\end{aligned}
$$

Next we use degenerate perturbation theory to calculate $E_{j}^{(k)}(g), j=0,1$. Applying the Cauchy integration formula to (95) and using the definitions of $f_{\beta, \mu}$ and $f_{\beta, \mu}^{\#}$, it follows that

$$
\begin{aligned}
R e \ll \phi_{k}^{0,1}, \tilde{\Sigma}_{3}^{k(2)} \phi_{k}^{0,1} \gg & =-\operatorname{Re} \ll \phi_{k}^{1,0}, \tilde{\Sigma}_{3}^{k(2)} \phi_{k}^{0,1} \gg \\
& = \pm \sum_{m \in \mathbf{Z}} \sum_{i=1}^{n} \mathcal{P} V \int_{\mathbf{R}} d u \frac{\left\|\hat{f}_{\beta_{i}, \mu_{i}, m(u, w)}\right\|_{\mathcal{B}}^{2}}{2 \omega_{0}-(k-m) \omega-u}, \\
I m \ll \phi_{k}^{0,1}, \tilde{\Sigma}_{3}^{k(2)} \phi_{k}^{0,1} \gg & =-I m \ll \phi_{k}^{1,0}, \tilde{\Sigma}_{3}^{k(2)} \phi_{k}^{0,1} \gg \\
& =-\pi \sum_{m \in \mathbf{Z}} \sum_{i=1}^{n}\left\|\hat{f}_{\beta_{i}, \mu_{i}, m}\left(2 \omega_{0}-(k-m) \omega, \omega\right)\right\|_{\mathcal{B}}^{2} .
\end{aligned}
$$


Therefore,

$$
E_{0,1}^{(k)}(g)=k \omega+g^{2} a_{0,1}+O\left(g^{4}\right),
$$

where $a_{0,1}$ are the eigenvalues of the $2 \times 2$ matrix

$$
-i \pi \sum_{m \in \mathbf{Z}} \sum_{i=1}^{n}\left\|\hat{f}_{\beta_{i}, \mu_{i}, m}\left(2 \omega_{0}-(k-m) \omega, \omega\right)\right\|_{\mathcal{B}}^{2}\left(\begin{array}{cc}
1 & -1 \\
-1 & 1
\end{array}\right) .
$$

By construction, $K_{g} e^{i k \omega t} \Omega=k \omega e^{i k \omega t} \Omega$ and $U(\theta) e^{i k \omega t} \Omega=e^{i k \omega t} \Omega$, so $\{k \omega: k \in$ $\mathbf{Z}\}$ are also isolated eigenvalues of $K_{g}^{*}(\theta), \in I^{-}(\delta)$. This can be seen by defining the spectral projections corresponding to the real isolated eigenvalues of $K_{g}(\theta)$, using the resolvent, and taking the adjoint to define the corresponding spectral projections for the real isolated eigenvalues of $K_{g}^{*}(\theta) .{ }^{12}$

The vector $\psi=\left(\begin{array}{l}1 \\ 1\end{array}\right)$ is the eigenvector corresponding the eigenvalue 0 of $\tilde{\Sigma}_{g,(k)}^{2}$. Hence,

$$
\begin{gathered}
E_{0}^{(k)}(g)=k \omega \\
E_{1}^{(k)}(g)=k \omega-2 \pi i g^{2} \sum_{m \in \mathbf{Z}} \sum_{i=1}^{n}\left\|\hat{f}_{\beta_{i}, \mu_{i}, m}\left(2 \omega_{0}-(k-m) \omega\right)\right\|_{\mathcal{B}}^{2}+\mathcal{O}\left(g^{4}\right)
\end{gathered}
$$

Note that due to assumption (A3), $\operatorname{Im} E_{j}^{(k)}<0$, for $j=1,2,3$, while $\operatorname{Im} E_{0}^{(k)}=$ $0 .{ }^{13}$ We have proven the following corollary to Theorem 4.3 .

Corollary 4.4. Suppose assumptions (A1)-(A3) hold. Then, for $\theta \in I^{-}(\kappa)$ and $|g|<g_{1} / 2$, where $g_{1}$ satisfies (81), $K_{g}^{*}(\theta)$ has infinitely many simple eigenvalues, $\{k \omega\}_{k \in Z}$, on the real axis, where $\omega=\frac{2 \pi}{\tau}$.

We will use the results of Theorem 4.3 and Corollary 4.4 to prove that the true state of the coupled system converges to a time-periodic state.

\footnotetext{
${ }^{12}$ Suppose $\alpha$ is an isolated and real eigenvalue of a closed operator $A$. Then the spectral projection corresponding to $\alpha$ is

$$
P=\frac{1}{2 \pi i} \oint_{\gamma_{\alpha}}(z-A)^{-1} d z
$$

where $\gamma_{\alpha}$ is a contour enclosing $\alpha$ only. Since $\alpha$ is real and isolated, it is also an eigenvalue of $A^{*}$ with corresponding projection $P^{*}$. (Using Cauchy's integration formula, one may readily verify that $A^{*} P^{*}=P^{*} A^{*}=\alpha P^{*}$ and that $\left(P^{*}\right)^{2}=P^{*}$.)

${ }^{13}$ Alternatively, one can use the Feshbach map (see Ref. 6) to compute the perturbation of the discrete spectrum of $K_{g}^{*}(\theta)$, which gives the same result.
} 


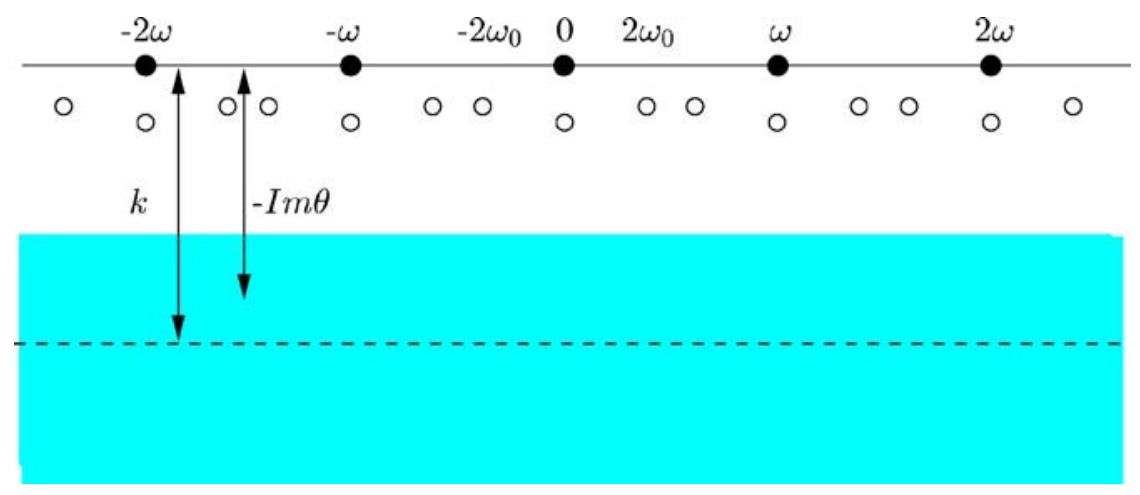

Fig. 1. Spectrum of $K_{g}^{*}(\theta)$. (color online)

\section{CONVERGENCE TO TIME-PERIODIC STATES}

The following theorem claims that, under suitable assumptions, the true state of the system converges to a time-periodic state with the period $\tau$ of the perturbation.

Choose $\kappa=\min \left(\delta, \frac{\pi}{\beta_{1}}, \ldots, \frac{\pi}{\beta_{n}}\right)$ (as in Sec. 4), define $\mathbf{h}^{\text {test }}:=D\left(e^{\kappa} \sqrt{p^{2}+1}\right)$, and let $\mathcal{O}^{\text {test }}, \mathcal{R}$ be the $*$-algebra generated by $b^{\#}(f), f \in \mathbf{h}^{\text {test }}$, and by $\mathbf{1}^{\mathcal{R}}$. Note that $\mathcal{O}^{\text {test }, \mathcal{R}}$ is norm-dense in $\mathcal{O}^{\mathcal{R}}$. We define a $*$-algebra

$$
\mathcal{C}:=\mathcal{O}^{\Sigma} \otimes \mathcal{O}^{\mathcal{R}_{1}, \text { test }} \otimes \cdots \otimes \mathcal{O}^{\mathcal{R}_{n, \text { test }}},
$$

which is dense in $\mathcal{O}$.

We make the following additional assumption.

(A4) The interaction Hamiltonian $V(t)$ belongs to $\mathcal{C}$, for $t \in \mathbf{R}$.

Let

$$
D:=\mathbf{1}^{\Sigma} \otimes \mathbf{1}^{\Sigma} \otimes e^{-\kappa \sqrt{A_{\mathcal{R}_{1}}^{2}+1}} \otimes \cdots \otimes e^{-k \sqrt{A_{\mathcal{R}_{n}}^{2}+1}},
$$

where $A_{\mathcal{R}_{i}}=d \Gamma\left(i \partial_{u_{i}}\right), i=1, \ldots, n$, is the second quantization of the generator of energy translations for the $i$ th reservoir. The operator $D$ is positive such that $D \Omega=\Omega$, and $\operatorname{Ran} D$ is dense in $\mathcal{H}$. This opertor will be used as a regulator in order to apply the complex deformation technique. We have the following theorem. We will show in the following theorem that the time-periodic state to which the real state of the coupled system converges is related to a zero-energy resonance state given by $\tilde{\Omega}_{g,(0)}:=(1 \otimes D) \tilde{P}_{g,(0)}(1 \otimes \Omega)$. 


\section{Theorem 5.1. (Convergence to time-periodic states).}

Assume assumptions (A1)-(A4) hold. Assume further that $a \in \mathcal{C}$. Then there is a constant $g_{1}>0$ satisfying (81), such that, for $|g|<g_{1} / 2$, the following holds

$$
\lim _{n \rightarrow \infty}\left\langle\Omega, \alpha_{g}^{n \tau+t}(a) \Omega\right\rangle=\left\langle\tilde{\Omega}_{g,(0)}, D^{-1} \alpha_{g}^{t}(a) \Omega\right\rangle,
$$

where $\tilde{\Omega}_{g,(0)}$ corresponds to the zero-energy resonance of the adjoint of the Floquet Liouvillean, $K_{g}^{*}$, and $D$ is given by (103).

Proof: First note that by using a Dyson series expansion, it follows from assumption (A4) and the fact that $a \in \mathcal{C}$ that $\alpha_{g}^{t}(a) \in \mathcal{C}$, and hence $\alpha_{g}^{t}(a) \Omega \in$ $\mathcal{D}\left(D^{-1}\right)$.

The remainder of the proof relies on the result of Theorem 4.3, Corollary 4.4, and equation (58) (Sec. 4). It follows from (58) and the time periodicity of $f \in \tilde{\mathcal{H}}=L^{2}([0, \tau]) \otimes \mathcal{H}$ that

$$
\left(e^{-i K_{g}^{*} n \tau} \mathbf{1} \otimes \Omega\right)(0)=\tilde{U}_{g}(n \tau, 0)(\mathbf{1} \otimes \Omega)(0)=\tilde{U}_{g}(n \tau, 0) \Omega .
$$

Let $\mathbf{1} \otimes \Omega=: \bar{\Omega} \in \tilde{\mathcal{H}}$, and $\bar{D}:=\mathbf{1} \otimes D$.

Without loss of generality, we assume that $\omega \equiv \frac{2 \pi}{\tau} \neq 2 \omega_{0}$; (if $\frac{2 \pi}{\tau}=2 \omega_{0}$, the state of the system typically oscillates between two resonance states until it finally converges to a time-periodic state; see remark 1). Using the dynamics on $C(\mathcal{O}, \Omega)$ and (105), it follows that

$$
\begin{aligned}
\lim _{n \rightarrow \infty}\left\langle\Omega, \alpha_{g}^{n \tau+t}(a) \Omega\right\rangle & =\lim _{n \rightarrow \infty}\left\langle\tilde{U}_{g}(n \tau, 0) \Omega, \alpha_{g}^{t}(a) \Omega\right\rangle \\
& =\lim _{n \rightarrow \infty}\left\langle\left(e^{-i K_{g}^{*} n \tau} \bar{\Omega}\right)(0), \alpha_{g}^{t}(a) \Omega\right\rangle
\end{aligned}
$$

Using the regulator $\bar{D}$ and complex spectral translation,

$$
\begin{aligned}
\lim _{n \rightarrow \infty}\left\langle\Omega, \alpha_{g}^{n \tau+t}(a) \Omega\right\rangle= & \lim _{n \rightarrow \infty}\left\langle\left(\bar{D} U(-\theta) e^{-i k_{g}^{*}(\theta) n \tau} U(\theta) \bar{D} \bar{\Omega}\right)(0), D^{-1} \alpha_{g}^{t}(a) \Omega\right\rangle \\
= & \lim _{n \rightarrow \infty}\left\langle\left(\bar{D} U(-\theta) \int_{-\infty}^{\infty} d u(u+i \eta\right.\right. \\
& \left.\left.\left.-K_{g}^{*}(\theta)\right)^{-1} e^{-i(u+i \eta) n \tau} \bar{\Omega}\right)(0), D^{-1} \alpha_{g}^{t}(a) \Omega\right\rangle .
\end{aligned}
$$

We split the above integration into two terms,

$$
\begin{gathered}
\lim _{n \rightarrow \infty}\left\langle\left(\bar{D} U(-\theta) \int_{-\infty}^{\infty} d u\left(u+i \eta-K_{g}^{*}(\theta)\right)^{-1} e^{-i(u+i \eta) n \tau} \bar{\Omega}(0), D^{-1} \alpha_{g}^{t}(a) \Omega\right\rangle\right. \\
=\lim _{n \rightarrow \infty}\left\langle\left(\bar{D} U(-\theta) \oint_{\gamma} d z\left(z-K_{g}^{*}(\theta)\right)^{-1} e^{-i z n \tau} \bar{\Omega}\right)(0), D^{-1} \alpha_{g}^{t}(a) \Omega\right\rangle+
\end{gathered}
$$




$$
\begin{aligned}
& +\lim _{n \rightarrow \infty}\left\langle\left(\bar{D} U(-\theta) \int_{-\infty}^{\infty} d u\left(u-i(\mu-\epsilon)-K_{g}^{*}(\theta)\right)^{-1} e^{-i(u-i(\mu-\epsilon)) n \tau} \bar{\Omega}\right)(0),\right. \\
& \left.D^{-1} \alpha_{g}^{t}(a) \Omega\right\rangle
\end{aligned}
$$

where $\eta>0,0<\epsilon<\mu$ and $\gamma$ is the contour enclosing the point spectrum of $K_{g}^{*}(\theta)$ only.

Using the results of Theorem 4.3 and Corollary 4.4, the first term converges to a time-periodic expression,

$$
\begin{aligned}
& \lim _{n \rightarrow \infty}\left\langle\left(\bar{D} U(-\theta) \oint_{\gamma} d z\left(z-K_{g}^{*}(\theta)\right)^{-1} e^{-i z n \tau} \bar{\Omega}\right)(0), D^{-1} \alpha_{g}^{t}(a) \Omega\right\rangle \\
& =\sum_{k \in \mathbf{Z}} \lim _{n \rightarrow \infty}\left\langle\left(\bar{D} U(-\theta) \tilde{S}_{g,(k)}^{-1}(\theta) e^{-i \tilde{\Sigma}_{g,(k)}(\theta) n \tau} \tilde{S}_{g,(k)}(\theta) \bar{\Omega}\right)(0), D^{-1} \alpha_{g}^{t}(a) \Omega\right\rangle \\
& \quad=\sum_{k \in \mathbf{Z}}\left\langle\left(\bar{D} \tilde{P}_{g,(k)}(\theta) \bar{\Omega}\right)(0), D^{-1} \alpha_{g}^{t}(a) \Omega\right\rangle
\end{aligned}
$$

Let

$$
\tilde{\Omega}_{g,(k)}:=\bar{D} \tilde{P}_{g,(k)}(\theta)\left(e^{i k \omega t} \otimes \Omega\right),
$$

and denote by $\ll \cdot, \cdot \gg$ the scalar product on $\tilde{\mathcal{H}}$. Then

$$
\bar{D} \tilde{P}_{g,(k)}(\theta) \bar{D}=\ll\left(e^{i k \omega t} \otimes \Omega\right), \cdot \gg \tilde{\Omega}_{g,(k)} .
$$

Therefore,

$$
\begin{aligned}
\bar{D} \tilde{P}_{g,(k)}(\theta) \bar{\Omega} & =\ll e^{i k \omega t} \otimes \Omega, \mathbf{1} \otimes \Omega \gg \tilde{\Omega}_{g,(k)} \\
& =\tilde{\Omega}_{g,(0)} \delta_{k, 0},
\end{aligned}
$$

where $\delta_{k, 0}$ is the Kronecker delta, and hence

$$
\sum_{k \in \mathbf{Z}}\left\langle\left(\bar{D} \tilde{P}_{g,(k)} \bar{\Omega}\right)(0), D^{-1} \alpha_{g}^{t}(a) \Omega\right\rangle=\left\langle\left(\tilde{\Omega}_{g,(0)}\right)(0), D^{-1} \alpha_{g}^{t}(a) \Omega\right\rangle,
$$

where $\tilde{\Omega}_{g,(0)}$ is the zero-energy resonance of the Floquet Liouvillean. The second term in (111) converges exponentially fast to zero since

$$
\begin{aligned}
& \left\langle\left(\bar{D} U(-\theta) \int_{-\infty}^{\infty} d u\left(u-i(\mu-\epsilon)-K_{g}^{*}(\theta)\right)^{-1} e^{-i(u-i(\mu-\epsilon)) n \tau} \bar{\Omega}\right)(0),\right. \\
& \left.D^{-1} \alpha_{g}^{t}(a) \Omega\right\rangle=O\left(e^{-\left(\mu-\epsilon^{\prime}\right) n \tau}\right),
\end{aligned}
$$

where $0<\epsilon^{\prime}<\epsilon<\mu$; (see Ref. 24, Chapter 19). 


\section{Remarks.}

(1) When $\omega=\frac{2 \pi}{\tau}=2 \omega_{0}$, the system exhibits the phenomenon of resonance: The state of the system oscillates between two resonances until it finally converges to the time periodic state corresponding to $\tilde{\Omega}_{g,(0)}$. This can be verified by a second order time-dependent perturbation theory calculation (see also Ref. 26).

(2) By a standard argument, the result of Theorem 5.1 can be extended to any initial state normal to $\omega$ (see for example, Refs. 16, 20, 21).

(3) Note that

$$
\left\langle\left(\tilde{\Omega}_{g,(0)}\right)(0), D^{-1} \alpha_{g}^{t}(a) \Omega\right\rangle=\left\langle\left(\tilde{\Omega}_{g,(k)}\right)(0), D^{-1} \alpha_{g}^{t}(a) e^{i k \omega t} \Omega\right\rangle,
$$

where $\tilde{\Omega}_{g,(k)}$ is the state corresponding to the kw-energy resonance of the adjoint of the Floquet Liouvillean. In other words, all kw-energy resonances belong to the same class of time-periodic states.

In the next section, we discuss the positivity of entropy production per cycle and Carnot's formulation of the second law of thermodynamics.

\section{POSITIVITY OF ENTROPY PRODUCTION}

We consider a small system coupled to two fermionic reservoirs at the same chemical potential $\mu$, yet at two different temperatures $\beta_{1}$ and $\beta_{2}$, with $\beta_{1}<$ $\beta_{2}$. Together with assumptions (A1)-(A4), we assume that the perturbation is differentiable in $t$, for $t>0$. The first reservoir acts as a heat source, and the second reservoir as a heat sink. We want to show that, after the true state of the system has converged to a time-periodic state, the entropy production per cycle is strictly positive. We first prove that the time-periodic state, which the true state of the system converges to, is not normal to the initial state. ${ }^{14}$

We introduce the standard Floquet Liouvillean,

$$
\tilde{K}_{g}:=-i \partial_{t}+\mathcal{L}_{g}(t)
$$

acting on the extended Hilbert space $L^{2}([0, \tau]) \otimes \mathcal{H}$, with periodic boundary conditions in $t$, where $\mathcal{L}_{g}(t)=\mathcal{L}_{0}+g V(t)-g J V(t) J$, is the standard Liouvillean. We study the spectrum of the standard Floquet Liouvillean using complex spectral translations. Since the proof of the following proposition is very similar to the analysis in Sec. 4, we only sketch the main steps of the proof.

\footnotetext{
${ }^{14}$ In this section, we will use standard results about von Neumann algebras and Tomita-Takesaki modular theory. We refer the reader to Ref. 7, chapters 2.4 and 2.5 for an exposition of these results.
} 
Proposition 6.1. Suppose assumptions (A1)-(A3) (Sec. 3) hold. Then there exists a positive constant $g_{2}$, such that, for $|g|<g_{2}$, the spectrum of the standard Floquet Liouvillean $\tilde{K}_{g}$ defined in (121), $\sigma\left(\tilde{K}_{g}\right)$ is absolutely continuous and

$$
\sigma\left(\tilde{K}_{g}\right)=\sigma_{a c}\left(\tilde{K}_{g}\right)=\boldsymbol{R}
$$

Sketch of proof.

Let $U(\theta)$ as in Sec. 4. We define the complex deformed standard Floquet Liouvillean by

$$
\begin{aligned}
\tilde{K}_{g}(\theta): & =U(\theta) \tilde{K}_{g} U(-\theta) \\
& =-i \partial_{t}+\mathcal{L}_{g}(t, \theta)
\end{aligned}
$$

where

$$
\mathcal{L}_{g}(t, \theta)=\mathcal{L}_{0}+\theta N+g V^{\mathrm{tot}}(t, \theta),
$$

and

$$
\begin{aligned}
V^{\mathrm{tot}}(t, \theta) & =U(\theta)(V(t)-J V(t) J) U(-\theta) \\
& =\sum_{i=1}^{n}\left\{\sigma_{-} \otimes \mathbf{1}^{\Sigma} \otimes a^{*}\left(f_{i, \beta_{i}, \mu}^{(\theta)}(t)\right)+\sigma_{+} \otimes \mathbf{1}^{\Sigma} \otimes a\left(f_{i, \beta_{i}, \mu}^{(\theta)}(t)\right)\right. \\
& \left.-i \mathbf{1}^{\Sigma} \otimes \sigma_{-} \otimes(-1)^{N_{i}} a^{*}\left(f_{i, \beta_{i}, \mu}^{\#(\theta)}(t)\right)-i \mathbf{1}^{\Sigma} \otimes \sigma_{+} \otimes(-1)^{N_{i}} a\left(f_{i, \beta_{i}, \mu}^{\#(\theta)}(t)\right)\right\} .
\end{aligned}
$$

It follows from assumption (A2) that $V^{\text {tot }}(t, \theta)$ is bounded for $0 \in I(\delta)$. Let

$$
\tilde{C}:=\sup _{t \in \mathbf{R}, \theta \in I(\delta)}\left\|V^{\text {tot }}(t, \theta)\right\| .
$$

For $\theta \in I^{-}(\kappa)$, choose $v$ such that $0>v>-\kappa$ and $-\kappa<\operatorname{Im} \theta<-(\kappa+|v|) / 2$. Choose $g_{2}>0$ such that

$$
g_{2} \tilde{C}<(k+v) / 4 .
$$

Then, using an argument which is similar to the proof of Theorem 4.3, one can show that, for $|g|<g_{2}$ the essential spectrum of $\tilde{K}_{g}(\theta)$ is contained in the halfplane $\{z \in \mathbf{C}: \operatorname{Im} z<v\}$, and that it discrete spectrum

$$
\sigma_{p p}\left(\tilde{K}_{g}\right)=\left\{\tilde{E}_{j}^{(k)}(g): k \in \mathbf{Z}, j=0, \ldots, 3\right\},
$$

where (to second order in perturbation theory)

$$
\tilde{E}_{2,3}^{(k)}(g)=k \omega \mp 2 \omega_{0} \mp g^{2} \sum_{m \in \mathbf{Z}} \sum_{i=1}^{2} \mathcal{P} V \int_{\mathbf{R}} d u \frac{\left\|\hat{f}_{\beta_{i}, \mu_{i}, m}(u, \omega)\right\|_{\mathcal{B}}^{2}}{2 \omega_{0}-(k-m) \omega-u}-
$$




$$
-i \pi g^{2} \sum_{m \in \mathbf{Z}} \sum_{i=1}^{2}\left\|\hat{f}_{\beta_{i}, \mu_{i}, m}\left(2 \omega_{0}-(k-m) \omega, \omega\right)\right\|_{\mathcal{B}}^{2}+\mathcal{O}\left(g^{4}\right),
$$

while

$$
\tilde{E}_{0,1}^{(k)}(g)=k \omega+g^{2} a_{0,1}+O\left(g^{4}\right),
$$

where $a_{0,1}$ are the eigenvalues of the $2 \times 2$ matrix

$$
\begin{aligned}
& -i \pi \sum_{m \in \mathbf{Z}} \sum_{i=1}^{2}\left\|\hat{f}_{\beta_{i}, \mu_{i}, m}\left(2 \omega_{0}-(k-m) \omega, \omega\right)\right\|_{\mathcal{B}}^{2} \\
& \times\left(\begin{array}{cc}
1 & -e^{-\beta_{i}\left(2 \omega_{0}-(k-m) \omega-\mu\right) / 2} \\
-e^{-\beta_{i}\left(2 \omega_{0}-(k-m) \omega-\mu\right) / 2} & 1
\end{array}\right) .
\end{aligned}
$$

Note that, to second order in the coupling $g$, the discrete spectrum is below the real axis. The claim of the theorem follows by noting that

$$
s-\lim _{\operatorname{Im} \theta \uparrow 0}\left(\tilde{K}_{g}(\theta)-z\right)^{-1}=\left(\tilde{K}_{g}(\operatorname{Re} \theta)-z\right)^{-1}
$$

for small real $g$ and large enough $\operatorname{Im} z$.

This result is sufficient to show that the time periodic state $\omega_{g, s}^{+}$defined in (9), Sec. 2, is not normal to the initial state $\omega$. Let $g_{3}:=\min \left\{g_{1} / 2, g_{2}\right\}$, where $g_{1}$ satisfies (81) (Sec. 4) and $g_{2}$ satisfies (125).

Theorem 6.2. Suppose assumptions (A1)-(A3) (Sec. 3) and (A4) (Sec. 5) hold. Then, for $|g|<g_{3}$, the time-periodic state $\omega_{g, s}^{+}$, for $s \in[0, \tau)$, does not belong to $\mathcal{N}_{\omega}$, ie, $\omega_{g, s}^{+}$is not normal with respect to $\omega$.

Proof: First note that under the assumptions of this theorem, the results of Proposition 6.1 hold. In particular, $\tilde{K}_{g}$ has no real eigenvalues.

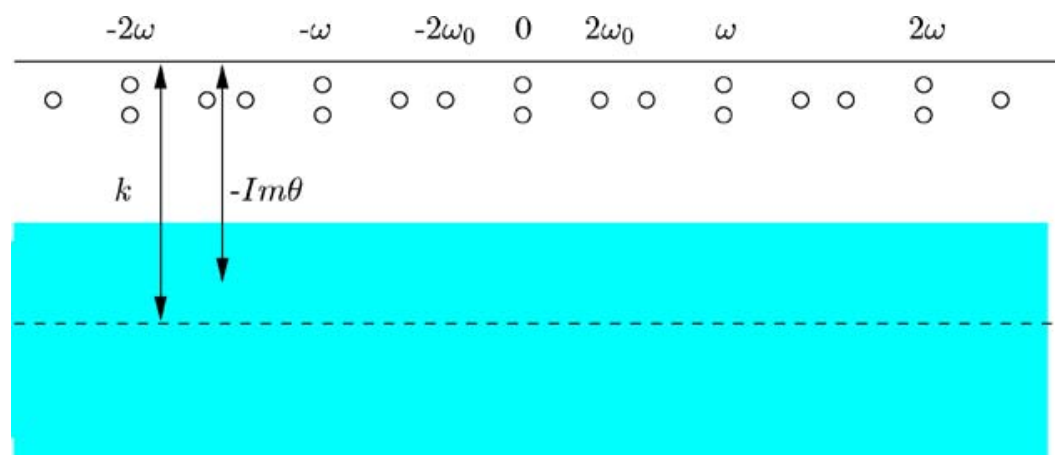

Fig. 2. Spectrum of $\tilde{K}_{g}(0)$. (color online) 
By construction,

$$
\omega_{g, s}^{+} \circ \alpha_{g}^{ \pm \tau}=\omega_{g, s}^{+} .
$$

Since we are assuming that $\omega_{g, s}^{+} \in \mathcal{N}_{\omega}$, there exists a unique vector $\Omega_{g, s}^{+}$in the natural positive cone $\mathcal{P}:=\{a J a \Omega: a \in \mathcal{M}\}$ associated to the pair $(\mathcal{M}, \omega)$, such that

$$
\omega_{g, s}^{+}(a)=\left\langle\Omega_{g, s}^{+}, a \Omega_{g, s}^{+}\right\rangle,
$$

for $a \in \mathcal{M} .{ }^{15}$ Now, (46), (128), and (129) imply that

$$
\left\langle\bar{U}_{g}(\tau, 0) \Omega_{g, s}^{+}, a \bar{U}_{g}(\tau, 0) \Omega_{g, s}^{+}\right\rangle=\left\langle\Omega_{g, s}^{+}, a \Omega_{g, s}^{+}\right\rangle,
$$

for $a \in \mathcal{M}$. Using the facts that

$$
\left[J, \bar{U}_{g}\left(t, t^{\prime}\right)\right]=0,
$$

where $J$ is the modular conjugation and $\bar{U}_{g}$ is the propagator generated by the standard Liouvillean, $J \Omega_{g, s}^{+}=\Omega_{g, s}^{+}$since $\Omega_{g, s}^{+} \in \mathcal{P}, J^{*}=J$, and $J \mathcal{M} J=$ $\mathcal{M}^{\prime}$, it follows that (130) also holds for a $a \in \mathcal{M}^{\prime}$. Furthermore, we know that $^{16}$

$$
\bar{U}_{g} \mathcal{P} \subset \mathcal{P},
$$

and that the linear span of $\mathcal{P}$ is dense in $\mathcal{H}$. Let $\mathrm{P}_{g, s}^{+}$be the orthogonal projection onto $\Omega_{g, s}^{+}$, then $\mathrm{P}_{g, s}^{+} \in \mathcal{M} \vee \mathcal{M}^{\prime}$. Moreover, since $\pi=\pi^{\Sigma} \otimes \pi^{\mathcal{R}_{1}} \otimes \cdots \otimes \pi^{\mathcal{R}_{n}}$, in the Araki-Wyss representation, $\mathcal{M}$ is a factor (of type $I I I_{1}$ see Ref. 2), ie, $\mathcal{M} \cap \mathcal{M}^{\prime}=\{\mathbf{C 1}\} .{ }^{17}$ Suppose that

$$
\bar{U}_{g}(\tau, 0) \Omega_{g, s}^{+}=c_{1} \Omega_{g, s}^{+}+c_{2} \Psi,
$$

where $\Psi \in \operatorname{Ran}\left(1-\mathrm{P}_{g, s}^{+}\right)$and $c_{1,2}$ are complex numbers to be determined. Choosing $a=\left(1-\mathrm{P}_{g, s}^{+}\right)$in (130) gives

$$
\left|c_{2}\right|^{2}=0
$$

Together with the fact that

$$
\left\langle\bar{U}_{g}(\tau, 0) \Omega_{g, s}^{+}, \bar{U}_{g}(\tau, 0) \Omega_{g, s}^{+},\right\rangle=\left\langle\Omega_{g, s}^{+}, \Omega_{g, s}^{+}\right\rangle=1,
$$

it follows that $\left|c_{1}\right|^{2}=1$. This implies that there exists $\lambda \in \mathbf{R}$ such that

$$
\bar{U}_{g}(\tau, 0) \Omega_{g, s}^{+}=e^{-i \lambda \tau} \Omega_{g, s}^{+} .
$$

\footnotetext{
${ }^{15}$ For a proof of this statement, see Ref. 7, Theorems 2.5.31 and 2.3.19.

${ }^{16}$ see Corollary 2.5 .32 in Ref. 7.

${ }^{17}$ Using the isomorphism between $\mathcal{F}^{\mathcal{R}_{1}}\left(L^{2}\right) \otimes \cdots \otimes \mathcal{F}^{\mathcal{R}_{n}}\left(L^{2}\right)$ and $\mathcal{F}\left(L_{\mathcal{R}_{1}}^{2} \otimes \cdots \otimes L_{\mathcal{R}_{n}}^{2}\right)$, one can proceed to show that $\mathcal{M}$ is a factor as in the case of the Araki-Wyss representation for a single reservoir of free fermions.
} 
For each fixed $s \in[0, \tau]$, we define

$$
\phi_{S}(t):=e^{i \lambda t} \bar{U}_{g}(t, 0) \Omega_{g, s}^{+},
$$

then $\phi_{s}$ is an eigenfunction of $\tilde{K}_{g}$ with eigenvalue $\lambda$. (This can be checked by looking at $\tilde{K}_{g} \phi_{s}$.) However, this is in contradiction with the result of Proposition 6.1, and hence $\omega_{g, s}^{+} \notin \mathcal{N}_{\omega}$.

We have the following result regarding the strict positivity of entropy production per cycle.

Theorem 6.3. (Positivity of entropy production) Suppose assumptions (A1) (A4) hold. Then the entropy production per cycle, after the state of the system has converged to a time-periodic state, is strictly positive, ie,

$$
\Delta E n t:=\int_{0}^{\tau} d t \omega_{g, t}^{+}\left(\delta_{\omega}(g V(t))\right)>0 .
$$

Proof: It follows from assumptions (A1)-(A4) and Theorem 5.1, that

$$
\sup _{T \in \mathbf{R}^{+}}\left|\int_{0}^{T} d t\left\{\omega_{g, t \bmod \tau}^{+}\left(\delta_{\omega}(g V(t))\right)-\omega \circ \alpha_{g}^{t}\left(\delta_{\omega}(g V(v))\right)\right\}\right|<\infty .
$$

Together with the result of Theorem 6.2, this implies that $\omega_{g, s}^{+}$satisfies the assumptions of Proposition 2.1, and the entropy production per cycle after the state of the coupled system has converged to a time-periodic state, is strictly positive.

Regarding the explicit computation of entropy production per cycle, (134), we would like to make the following remark. Since it follows from Theorem 4.3 that $\tilde{\Omega}_{g,(0)}$ is analytic in $g$ for $g<g_{1} / 2$, one can expand $\omega_{g, s}^{+}$to any order in the coupling $g$, and compute an explicit expression for $\Delta E n t$ given in (134) up to this order in the coupling constant; (see also Refs. 20, 21 for a discussion of a perturbative approach for calculating entropy production in nonequilibrium steady-states).

\section{APPENDIX: GLUED HILBERT SPACE REPRESENTATION}

We want to show that

$$
\mathcal{F}\left(L^{2}\left(\mathbf{R}^{+} ; \mathcal{B}\right)\right) \otimes \mathcal{F}\left(L^{2}\left(\mathbf{R}^{+} ; \mathcal{B}\right)\right) \cong \mathcal{F}\left(L^{2}(\mathbf{R} ; \mathcal{B})\right) .
$$


Let $\Omega$ be the vacuum state in the fermionic Fock space $\mathcal{F}\left(L^{2}\left(\mathbf{R}^{+} ; \mathcal{B}\right)\right)$. For fermionic creation/annihilation operators on $\mathcal{F}\left(L^{2}\left(\mathbf{R}^{+} ; \mathcal{B}\right)\right)$,

$$
b^{\#}(f):=\int m(u) d u d \sigma f(u, \sigma) b^{\#}(u, \sigma), f \in L^{2}\left(\mathbf{R}^{+} ; \mathcal{B}\right),
$$

define the creation/annihilation operators on $\mathcal{F}\left(L^{2}\left(\mathbf{R}^{+} ; \mathcal{B}\right)\right) \otimes \mathcal{F}\left(L^{2}\left(\mathbf{R}^{+} ; \mathcal{B}\right)\right)$ as

$$
\begin{aligned}
& b_{l}^{\#}(f):=b^{\#}(f) \otimes \mathbf{1} ; \\
& b_{r}^{\#}(f):=(-1)^{N} \otimes b^{\#}(\bar{f}),
\end{aligned}
$$

where ${ }^{-}$corresponds to complex conjugation. Note that $b_{1}$ and $b_{r}$ anti-commute. Let $\tilde{a}$ and $\tilde{a}^{*}$ be the annihilation and creation operators on the fermionic Fock space $\mathcal{F}\left(L^{2}\left(\mathbf{R}^{+} ; \mathcal{B}\right) \oplus\left(L^{2}\left(\mathbf{R}^{+} ; \mathcal{B}\right)\right)\right.$, such that they satisfy the usual CAR, and let $\tilde{\Omega}$ be the vacuum state in $\mathcal{F}\left(L^{2}\left(\mathbf{R}^{+} ; \mathcal{B}\right) \oplus\left(L^{2}\left(\mathbf{R}^{+} ; \mathcal{B}\right)\right)\right.$. An isomorphism between $\mathcal{F}\left(L^{2}\left(\mathbf{R}^{+} ; \mathcal{B}\right)\right) \otimes \mathcal{F}\left(L^{2}\left(\mathbf{R}^{+} ; \mathcal{B}\right)\right)$ and $\mathcal{F}\left(L^{2}\left(\mathbf{R}^{+} ; \mathcal{B}\right) \oplus\left(L^{2}\left(\mathbf{R}^{+} ; \mathcal{B}\right)\right)\right.$ follows by the identification

$$
\begin{aligned}
& b_{l}^{\#}(f) \cong \tilde{a}^{\#}((f, 0)), \\
& b_{r}^{\#}(g) \cong \tilde{a}^{\#}((0, g)), \\
& \Omega \otimes \Omega \cong \tilde{\Omega} .
\end{aligned}
$$

Now we claim that $\mathcal{F}\left(L^{2}\left(\mathbf{R}^{+} ; \mathcal{B}\right) \oplus\left(L^{2}\left(\mathbf{R}^{+} ; \mathcal{B}\right)\right)\right.$ is isomorphic to $\mathcal{F}\left(L^{2}(\mathbf{R}, d u ; \mathcal{B})\right)$. For $\phi, \psi \in \mathbf{R}$, consider the mapping

$$
j_{\phi, \psi}: L^{2}\left(\mathbf{R}^{+} ; \mathcal{B}\right) \oplus L^{2}\left(\mathbf{R}^{+} ; \mathcal{B}\right) \ni(f, g) \rightarrow h \in L^{2}(\mathbf{R} d u ; \mathcal{B})
$$

such that

$$
h(u, \sigma):=\left\{\begin{array}{l}
e^{i \phi} \sqrt{m(u)} f(u, \sigma), \quad u \geq 0 \\
e^{i \psi} \sqrt{m(|u|)} g(|u|, \sigma), \quad u<0
\end{array} .\right.
$$

This mapping is an isometry, since

$$
\begin{aligned}
\|h\|_{L^{2}(\mathbf{R}, d u ; \mathcal{B})}^{2} & =\|(f, g)\|_{L^{2}\left(\mathbf{R}^{+} ; \mathcal{B}\right) \otimes L^{2}\left(\mathbf{R}^{+} ; \mathcal{B}\right)}^{2} \\
& =\int_{\mathbf{R}^{+} ; \mathcal{B}} d u d \sigma m(u)|f(u, \sigma)|^{2}+\int_{\mathbf{R}^{+} ; \mathcal{B}} d u d \sigma m(u)|g(u, \sigma)|^{2} \\
& =\|f\|_{L^{2}\left(\mathbf{R}^{+} ; \mathcal{B}\right)}^{2}+\|g\|_{L^{2}\left(\mathbf{R}^{+} ; \mathcal{B}\right)}^{2}
\end{aligned}
$$

Moreover, the mapping $j_{\phi, \psi}$ is an isomorphism, since, for given $h \in$ $L^{2}(\mathbf{R} ; \mathcal{B})$, there exists a mapping $j_{\phi, \psi}^{-1}: h \rightarrow(f, g) \in L^{2}\left(\mathbf{R}^{+} ; \mathcal{B}\right) \otimes L^{2}\left(\mathbf{R}^{+}, \mathcal{B}\right)$, 
such that

$$
\begin{aligned}
& f(u, \sigma):=\frac{e^{-i \phi}}{\sqrt{m(u)}} h(u, \sigma), \quad u>0, \\
& g(u, \sigma):=\frac{e^{-i \psi}}{\sqrt{m(|u|)}} h(|u|, \sigma), \quad u<0 .
\end{aligned}
$$

\section{ACKNOWLEDGMENT}

W.A.S. would like to thank Gian Michele Graf for enjoyable discussions.

\section{REFERENCES}

1. H. Araki, Relative Hamiltonian for faithful normal states of a von Neumann algebra. Publ. Res. Inst. Math. Sci. Kyoto Univ. 9:165 (1973).

2. H. Araki and W. Wyss, Representations of canonical anticommutation relations. Helv. Phys. Acta 37:136 (1964).

3. W. Abou Salem, Nonequilibrium quantum statistical mechanics and thermodynamics. ETH-Diss. 16187 (2005)

4. W. Abou Salem and J. Fröhlich, Status of the fundamental laws of thermodynamics, in preparation.

5. W. Abou Salem and J. Fröhlich, Adiabatic theorems and reversible isothermal processes. Lett. Math. Phys. 72:153-163 (2005).

6. V. Bach, J. Fröhlich, and I. M. Sigal, Return to Equilibrium, J. Math. Phys. 41(6):3985-4061 (2000).

7. O. Bratteli and D. Robinson, Operator Algebras and Quantum Statistical Mechanics 1,2, Texts and Monographs in Physics (Springer-Verlag, Berlin, 1987).

8. M. J. Donald, Relative Hamiltonians which are not bounded from above. J. Func. Anal. 91:143 (1990).

9. J. Dereziński, V. Jaksić, and C.-A. Pillet, Perturbation theory of $W^{*}$-dynamics, Liouvilleans, and KMS-states, Rev. Math. Phys. 15:447-489 (2003).

10. J. Fröhlich, M. Merkli, S. Schwarz, and D. Ueltschi, Statistical mechanics of thermodynamic processes, A Garden of Quanta (World Sci. Publishing, River Edge, New Jersey, 2003, pp. 345 363).

11. J. S. Howland, Stationary scattering theory for time dependent Hamiltonians. Math. Ann. 207:315335 (1974).

12. W. Hunziker and C.-A. Pillet, Degenerate asymptotic perturbation theory. Commun. Math. Phys. 90:219 (1983).

13. W. Hunziker, Notes on asymptotic perturbation theory for Schrödinger eigenvalue problems, Helv. Phys. Acta 61:257-304 (1988).

14. V. Jaksić and C. A. Pillet, On a Model for Quantum Friction II. Fermi's Golden Rule and Dynamics at Positive Temperature. Commun. Math. Phys. 176:619-644 (1996).

15. V. Jaksić and C. A. Pillet, On a Model for Quantum Friction III. Ergodic Properties of the SpinBoson System. Commun. Math. Phys. 178:627-651 (1996).

16. V. Jaksić and C.-A. Pillet, Non-equilibrium steady states of finite quantum systems coupled to thermal reservoirs. Commun. Math. Phys. 226:131-162 (2002). 
17. V. Jaksić and C.-A. Pillet, A note on the entropy production formula. Advances in Differential Equations and Mathematical Physics, pp. 175-180, Contemp. Math. 327, American Mathematical Society, Providence, RI, 2003.

18. T. Kato, Perturbation Theory for Linear Operators, (Springer, Berlin, 1980).

19. T. Kato, Linear evolution equations of hyperbolic type. I.J. Fac. Sci. Univ. Tokyo Sect. IA 17:241258 (1970).

20. M. Merkli, M. Mück, and I. M. Sigal, Instability of equilibrium states for coupled heat reservoirs at different temperatures, [axiv:math-ph/0508005].

21. M. Merkli, M. Mück, and I. M. Sigal, Theory of nonequilibrium stationary states as a theory of resonances. Existence and properties of NESS, [arxiv:math-ph/0603006].

22. W. Pusz and S. L. Woronowicz, Passive states and KMS states for general quantum systems. Commun. Math. Phys. 58:273-290 (1978).

23. M. Reed and B. Simon. Methods of Modern Mathematical Physics, Vol. I (Functional Analysis), Vol. II (Fourier Analysis, Self-Adjointness), Academic Press, New York 1975.

24. W. Rudin, Real and Complex Analysis, 3rd ed., (Mc-Graw-Hill, New York, 1987).

25. K. Yajima, Scattering theory for Schrödinger equations with potentials periodic in time. J. Math. Soc. Jpn. 29:729-743 (1977).

26. K. Yajima, Resonances for AC-Stark effect. Commun. Math. Phys. 87:331-352 (1982).

27. K. Yosida, Functional Analysis, 6th ed., (Springer-Verlag, Berlin, 1998). 